\title{
How adaptation currents change threshold, gain, and variability of
}

\section{neuronal spiking}

\author{
Josef Ladenbauer, ${ }^{1,2}$ Moritz Augustin, ${ }^{1,2}$ and Klaus Obermayer ${ }^{1,2}$ \\ ${ }^{1}$ Neural Information Processing Group, Technische Universität Berlin, Berlin, Germany; and ${ }^{2}$ Bernstein Center \\ for Computational Neuroscience Berlin, Berlin, Germany
}

Submitted 19 August 2013; accepted in final form 25 October 2013

Ladenbauer J, Augustin M, Obermayer K. How adaptation currents change threshold, gain, and variability of neuronal spiking. $J$ Neurophysiol 111: 939-953, 2014. First published October 30, 2013; doi:10.1152/jn.00586.2013.-Many types of neurons exhibit spike rate adaptation, mediated by intrinsic slow $\mathrm{K}^{+}$currents, which effectively inhibit neuronal responses. How these adaptation currents change the relationship between in vivo like fluctuating synaptic input, spike rate output, and the spike train statistics, however, is not well understood. In this computational study we show that an adaptation current that primarily depends on the subthreshold membrane voltage changes the neuronal input-output relationship (I-O curve) subtractively, thereby increasing the response threshold, and decreases its slope (response gain) for low spike rates. A spike-dependent adaptation current alters the I-O curve divisively, thus reducing the response gain. Both types of an adaptation current naturally increase the mean interspike interval (ISI), but they can affect ISI variability in opposite ways. A subthreshold current always causes an increase of variability while a spike-triggered current decreases high variability caused by fluctuation-dominated inputs and increases low variability when the average input is large. The effects on I-O curves match those caused by synaptic inhibition in networks with asynchronous irregular activity, for which we find subtractive and divisive changes caused by external and recurrent inhibition, respectively. Synaptic inhibition, however, always increases the ISI variability. We analytically derive expressions for the I-O curve and ISI variability, which demonstrate the robustness of our results. Furthermore, we show how the biophysical parameters of slow $\mathrm{K}^{+}$conductances contribute to the two different types of an adaptation current and find that $\mathrm{Ca}^{2+}$-activated $\mathrm{K}^{+}$currents are effectively captured by a simple spike-dependent description, while muscarine-sensitive or $\mathrm{Na}^{+}$-activated $\mathrm{K}^{+}$currents show a dominant subthreshold component.

adaptation; gain modulation; Hodgkin-Huxley-like model; integrateand-fire model; spike train

ADAPTATION IS A WIDESPREAD phenomenon in nervous systems, providing flexibility to function under varying external conditions. At the single neuron level, this can be observed as spike rate adaptation, a gradual decrease in spiking activity following a sudden increase in stimulus intensity. This type of intrinsic inhibition, in contrast to the one caused by synaptic interaction, is typically mediated by slowly decaying somatic $\mathrm{K}^{+}$currents, which accumulate when the membrane voltage increases. A number of slow $\mathrm{K}^{+}$currents with different activation characteristics have been identified. Muscarine-sensitive (Brown and Adams 1980; Adams et al. 1982) or $\mathrm{Na}^{+}$-dependent $\mathrm{K}^{+}$channels activate at subthreshold voltage values (Schwindt et al. 1989; Kim and McCormick 1998), whereas $\mathrm{Ca}^{2+}$-dependent

Address for reprint requests and other correspondence: J. Ladenbauer, Technische Universität Berlin, Neural Information Processing Group, Marchstr. 23, 10587 Berlin, Germany (e-mail: j1@ ni.tu-berlin.de).
$\mathrm{K}^{+}$channels activate at higher, suprathreshold values (Brown and Griffith 1983; Madison and Nicoll 1984; Schwindt et al. 1992). Such adaptation currents, for example, mediate frequency selectivity of neurons (Fuhrmann et al. 2002; Benda et al. 2005; Ellis et al. 2007), where the preferred frequency depends on the current activation type (Deemyad et al. 2012). They promote network synchronization (Sanchez-Vives and McCormick 2000; Augustin et al. 2013; Ladenbauer et al. 2013) and are likely involved in the attentional modulation of neuronal response properties by acetylcholine (Herrero et al. 2008; Soma et al. 2012; McCormick 1992). It has been hypothesized that these complex effects are produced by changing the relationship between synaptic input and spike rate output (I-O curve) (Deemyad et al. 2012; Benda and Herz 2003; Soma et al. 2012; Reynolds and Heeger 2009). For example, changing the I-O curve of a neuron subtractively sharpens stimulus selectivity, whereas a divisive change downscales the neuronal response but preserves selectivity (see Wilson et al. 2012 in the context of synaptic inhibition). It was also suggested that adaptation currents affect the neural code via their effect on the interspike interval (ISI) statistics (Prescott and Sejnowski 2008). So far, the effects of adaptation currents on I-O curves have been studied considering constant current inputs disregarding input fluctuations (Prescott and Sejnowski 2008; Deemyad et al. 2012) and it has remained unclear how different types of an adaptation current affect ISI variability. Therefore, in this contribution we systematically examine how voltage-dependent subthreshold and spike-dependent adaptation currents change neuronal I-O curves as well as the ISI distribution for typical in vivo like input statistics and how the biophysical parameters of slow $\mathrm{K}^{+}$conductances contribute to the two types of adaptation current.

We address these questions by studying spike rates and ISI distributions of model neurons with subthreshold and spiketriggered adaptation currents, subject to fluctuating in vivo like inputs, and we compare the results to those induced by synaptic inhibition. Specifically, we use the adaptive exponential integrate-and-fire (aEIF) neuron model (Brette and Gerstner 2005), which has been shown to perform well in predicting the subthreshold properties (Badel et al. 2008) and spiking activity (Jolivet et al. 2008; Pospischil et al. 2011) of cortical neurons. To analytically demonstrate the changes of I-O curves and ISI variability we derive explicit expressions for these properties based on the simpler perfect integrate-and-fire neuron model (see, e.g., Gerstein and Mandelbrot 1964) with adaptation (aPIF). Finally, using a detailed conductance-based neuron model we quantify the subthreshold and spike-triggered com- 
ponents of various slow $\mathrm{K}^{+}$currents and compare the effects of specific $\mathrm{K}^{+}$channels on the I-O curve and ISI variability.

\section{MATERIALS AND METHODS}

aEIF neuron with noisy input current. We consider an aEIF model neuron receiving synaptic input currents. The subthreshold dynamics of the membrane voltage $V$ is given by

$$
C \frac{d V}{d t}=I_{\text {ion }}(V)+I_{\mathrm{syn}}(t)
$$

where the capacitive current through the membrane with capacitance $C$ equals the sum of ionic currents $I_{\text {ion }}$ and the synaptic current $I_{\text {syn }}$. Three ionic currents are taken into account,

$$
I_{\text {ion }}(V):=-g_{\mathrm{L}}\left(V-E_{\mathrm{L}}\right)+g_{\mathrm{L}} \Delta_{\mathrm{T}} \exp \left(\frac{V-V_{\mathrm{T}}}{\Delta_{\mathrm{T}}}\right)-w .
$$

The first term on the right-hand side describes the leak current with conductance $g_{\mathrm{L}}$ and reversal potential $E_{\mathrm{L}}$. The exponential term with threshold slope factor $\Delta_{\mathrm{T}}$ and effective threshold voltage $V_{\mathrm{T}}$ approximates the fast $\mathrm{Na}^{+}$current at spike initiation, assuming instantaneous activation of $\mathrm{Na}^{+}$channels (Fourcaud-Trocmé et al. 2003). $w$ Is the adaptation current that reflects a slow $\mathrm{K}^{+}$current. It evolves according to

$$
\tau_{w} \frac{d w}{d t}=a\left(V-E_{w}\right)-w
$$

with adaptation time constant $\tau_{w}$. Its strength depends on the subthreshold membrane voltage via conductance $a$. $E_{w}$ denotes its reversal potential. When $V$ increases beyond $V_{\mathrm{T}}$, a spike is generated due to the exponential term in $E q$. 2. The downswing of the spike is not explicitly modeled, instead, when $V$ reaches a value $V_{\mathrm{s}} \geq V_{\mathrm{T}}$, the membrane voltage is reset to a lower value $V_{\mathrm{r}}$. At the same time, the adaptation current $w$ is incremented by a value of $b$, implementing the mechanism of spike-triggered adaptation. Immediately after the reset, $V$ and $w$ are clamped for a refractory period $T_{\text {ref }}$, and subsequently governed again by Eqs. 1-3.

The aEIF model can reproduce a wide range of neuronal subthreshold dynamics (Touboul and Brette 2008) and spike patterns (Naud et al. 2008). We selected the following parameter values to model cortical neurons: $C=1 \mu \mathrm{F} / \mathrm{cm}^{2}, g_{\mathrm{L}}=0.05 \mathrm{mS} / \mathrm{cm}^{2}, E_{\mathrm{L}}=-65 \mathrm{mV}$, $\Delta_{\mathrm{T}}=1.5 \mathrm{mV}, V_{\mathrm{T}}=-50 \mathrm{mV}, \tau_{w}=200 \mathrm{~ms}, E_{w}=-80 \mathrm{mV}, V_{\mathrm{s}}=$ $-40 \mathrm{mV}, V_{\mathrm{r}}=-70 \mathrm{mV}$, and $T_{\text {ref }}=1.5 \mathrm{~ms}$ (Badel et al. 2008; Destexhe 2009; Wang et al. 2003). The adaptation parameters $a$ and $b$ were varied within reasonable ranges, $a \in[0,0.06] \mathrm{mS} / \mathrm{cm}^{2}, b \in[0$, $0.3] \mu \mathrm{A} / \mathrm{cm}^{2}$.

The synaptic input consists of a mean $\mu(t)$ and a fluctuating part given by a Gaussian white noise process $\eta(t)$ with $\delta$-autocorrelation and standard deviation $\sigma(t)$,

$$
I_{\text {syn }}(t)=C[\mu(t)+\sigma(t) \eta(t)] .
$$

Equation 4 describes the total synaptic current received by $K_{\mathcal{E}}$ excitatory and $K_{\mathcal{I}}$ inhibitory neurons, which produce instantaneous postsynaptic potentials (PSPs) $J_{\mathcal{E}}>0$ and $J_{\mathcal{I}}<0$, respectively. For synaptic events (i.e., presynaptic spike times) generated by independent Poisson processes with rates $r_{\mathcal{E}}(t)$ and $r_{\mathcal{I}}(t)$, the infinitesimal moments $\mu(t)$ and $\sigma(t)$ are expressed as

$$
\begin{aligned}
& \mu(t)=J_{\mathcal{E}} K_{\mathcal{E}} r_{\mathcal{E}}(t)+J_{\mathcal{I}} K_{\mathcal{I}} r_{\mathcal{I}}(t), \\
& \sigma(t)^{2}=J_{\mathcal{E}}^{2} K_{\mathcal{E}} r_{\mathcal{E}}(t)+J_{\mathcal{I}}^{2} K_{\mathcal{I}} r_{\mathcal{I}}(t),
\end{aligned}
$$

assuming large numbers $K_{\mathcal{E}}, K_{\mathcal{I}}$, and small magnitudes of $J_{\mathcal{E}}, J_{\mathcal{I}}$ (Tuckwell 1988; Renart et al. 2004; Destexhe and Rudolph-Lilith 2012). This diffusion approximation well describes the activity in many cortical areas (Shadlen and Newsome 1998; Destexhe et al. 2003; Compte et al. 2003; Maimon and Assad 2009). The parameter values were $J_{\mathcal{E}}=0.15 \mathrm{mV}, J_{\mathcal{I}}=-0.45 \mathrm{mV}, K_{\mathcal{E}}=2000, K_{\mathcal{I}}=500$, and $r_{\mathcal{E}}, r_{\mathcal{I}}$ were varied in $[0,50] \mathrm{Hz}$. In addition, we directly varied $\mu$ and $\sigma$ over a wide range of biologically plausible values.

Membrane voltage distribution and spike rate. In the following we describe how we obtain the distribution of the membrane voltage $p(V$, $t$ ) and the instantaneous spike rate $r(t)$ of a single neuron at time $t$ for a large number $N$ of independent trials. Note that by trial we refer to a solution trajectory of the system of stochastic differential equations (Eqs. 1-4) for a realization of $\eta(t)$.

First, to reduce computational demands and enable further analysis, we replace the adaptation current $w$ in Eqs. 2 and 3 by its average over trials, $\bar{w}(t):=1 / N \sum_{i=1}^{N} w_{i}(t)$, where $i$ is the trial index (Gigante et al. 2007a). Neglecting the variance of $w$ across trials is valid under the assumption that the dynamics of the adaptation current is substantially slower than that of the membrane voltage, which is supported by empirical observations (Brown and Adams 1980; Sanchez-Vives and McCormick 2000; Sanchez-Vives et al. 2000; Stocker 2004). The instantaneous spike rate at time $t$ can be estimated by the average number of spikes in a small interval $[t, t+\Delta t]$,

$$
r_{\Delta t}(t):=\frac{1}{N \Delta t} \sum_{i=1}^{N} \int_{t}^{t+\Delta t} \sum_{k} \delta\left(s-t_{i}^{k}\right) d s,
$$

where $\delta$ is the delta function and $t_{i}^{k}$ denotes the $k$-th spike time in trial $i$. In the limit $N \rightarrow \infty, \Delta t \rightarrow 0$, the probability density $p(V, t)$ obeys the Fokker-Planck equation (Risken 1996; Tuckwell 1988; Renart et al. 2004),

$$
\frac{\partial}{\partial t} p(V, t)+\frac{\partial}{\partial V} q(V, t)=0,
$$

with probability flux $q(V, t)$ given by

$$
q(V, t):=\left(\frac{I_{\text {ion }}(V ; \bar{w})}{C}+\mu(t)\right) p(V, t)-\frac{\sigma(t)^{2}}{2} \frac{\partial}{\partial V} p(V, t) .
$$

$I_{\text {ion }}(V ; \bar{w})$ denotes the sum of ionic currents (cf. Eq. 2) where $w$ is replaced by the average adaptation current $\bar{w}$, which evolves according to

$$
\tau_{w} \frac{d \bar{w}}{d t}=a\left(\langle V\rangle_{p(V, t)}-E_{w}\right)-\bar{w}+\tau_{w} b r(t) .
$$

$\langle\cdot\rangle_{p}$ indicates the average with respect to the probability density $p$ (Brunel et al. 2003; Gigante et al. 2007b). To account for the reset of the membrane voltage, the probability flux at $V_{\mathrm{s}}$ is reinjected at $V_{\mathrm{r}}$ after the refractory period has passed, i.e.,

$$
\lim _{V \searrow V_{\mathrm{r}}} q(V, t)-\lim _{V \nearrow V_{\mathrm{r}}} q(V, t)=q\left(V_{s}, t-T_{\mathrm{ref}}\right) .
$$

The boundary conditions for this system are reflecting for $V \rightarrow-\infty$ and absorbing for $V=V_{\mathrm{s}}$,

$$
\lim _{V \rightarrow-\infty} q(V, t)=0, \quad p\left(V_{\mathrm{s}}, t\right)=0,
$$

and the (instantaneous) spike rate is obtained by the probability flux at $V_{\mathrm{s}}$,

$$
r(t)=q\left(V_{\mathrm{s}}, t\right) .
$$

Note that $p(V, t)$ only reflects the proportion of trials where the neuron is not refractory at time $t$, given by $P(t)=\int_{-\infty}^{V_{\mathrm{s}}} p(v, t) d v[<1$ for $T_{\text {ref }}>0$ and $r(t)>0$ ]. The total probability density that the membrane voltage is $V$ at time $t$ is given by $p(V, t)+p_{\text {ref }}(V, t)$, with refractory density $p_{\text {ref }}(V, t)=[1-P(t)] \delta\left(V-V_{\mathrm{r}}\right)$. Since $p(V, t)$ does not integrate to unity in general, the average in $E q .10$ is calculated as $\langle V\rangle_{p(V, t)}=\int_{-\infty}^{V_{\mathrm{s}}} v p(v, t) d v / P(t)$. The dynamics of the average adaptation current $\bar{w}(t)$ reflecting the nonrefractory proportion of trials is well captured by $E q .10$ as long as $T_{\text {ref }}$ is small compared with $\tau_{w}$. In this (physiologically plausible) case $\bar{w}(t)$ can be considered 
equal to the average adaptation current over the refractory proportion of trials.

Steady-state spike rate. We consider the membrane voltage distribution of an aEIF neuron with noisy synaptic input, described by the Eqs. $8-13$, has reached its steady-state $p_{\infty} . p_{\infty}$ obeys $\partial p_{\infty}(V) / \partial t=0$ or equivalently,

$$
\frac{\partial}{\partial V} q_{\infty}(V)=0
$$

with steady-state probability flux $q_{\infty}$ given by

$$
q_{\infty}(V)=\left(\frac{I_{\text {ion }}(V ; \bar{w})}{C}+\mu\right) p_{\infty}(V)-\frac{\sigma^{2}}{2} \frac{\partial}{\partial V} p_{\infty}(V),
$$

subject to the reset condition,

$$
\lim _{V \unlhd V_{\mathrm{r}}} q_{\infty}(V)-\lim _{V \nearrow V_{\mathrm{r}}} q_{\infty}(V)=q_{\infty}\left(V_{\mathrm{s}}\right),
$$

and the boundary conditions,

$$
\lim _{V \rightarrow-\infty} q_{\infty}(V)=0, \quad p_{\infty}\left(V_{\mathrm{s}}\right)=0 .
$$

The steady-state spike rate is given by $r_{\infty}=q_{\infty}\left(V_{\mathrm{s}}\right)$ and the steadystate mean adaptation current reads $\bar{w}_{\infty}=a\left(\langle V\rangle_{\infty}-E_{w}\right)+\tau_{w} b r_{\infty}$. We multiply both sides of $E q .14$ by $V$ and integrate over the interval $(-\infty$, $V_{\mathrm{s}}$ ], assuming that $p_{\infty}(V)$ tends sufficiently quickly toward zero for $V \rightarrow-\infty$ (Brunel 2000; Brunel et al. 2003), to obtain an equation that relates the steady-state spike rate and mean membrane voltage,

$$
r_{\infty}=\frac{\mu_{a}-g_{\mathrm{L}}\left[\langle V\rangle_{\infty}-E_{\mathrm{L}}+\Delta_{\mathrm{T}}\left\langle\exp \left(\frac{V-V_{\mathrm{T}}}{\Delta_{\mathrm{T}}}\right)\right\rangle_{\infty}\right] / C}{\Delta V+\tau_{w} b / C},
$$

where $\mu_{a}:=\mu-a\left(\langle V\rangle_{\infty}-E_{w}\right) / C, \Delta V:=V_{\mathrm{s}}-V_{\mathrm{r}}$ (here and in the following) and $\langle\cdot\rangle_{\infty}$ denotes the average with respect to the density $p_{\infty}(V)$. The spike rate $r_{\infty}$ is given by Eq. 18 only for nonnegative values of the numerator (i.e., $\mu_{a}-g_{\mathrm{L}}[\ldots] / C \geq 0$ ); otherwise, $r_{\infty}$ is defined to be zero. For simplicity, the refractory period $T_{\text {ref }}$ is omitted here. Note that the steady-state spike rate for $T_{\text {ref }} \neq 0$ can be calculated as $r_{\infty} /\left(1+r_{\infty} T_{\text {ref }}\right)$. We cannot express $p_{\infty}(V)$ explicitly and thus the expressions for the averages with respect to $p_{\infty}(V)$ in $E q .18$ are not known. However, in the case $g_{\mathrm{L}}=0$, which simplifies the aEIF model to the aPIF model, an explicit expression for $\langle V\rangle_{\infty}$ can be derived. We multiply $E q .14$ by $V^{2}$ and integrate over $\left(-\infty, V_{\mathrm{s}}\right]$ on both sides [assuming again that $p_{\infty}(V)$ quickly tends to zero for $V \rightarrow$ $-\infty]$ to obtain

$$
\langle V\rangle_{\infty}=\frac{1}{2 a}\left[A+a \frac{V_{\mathrm{s}}+V_{\mathrm{r}}}{2}-\sqrt{\left(A-a \frac{V_{\mathrm{s}}+V_{\mathrm{r}}}{2}\right)^{2}+B}\right],
$$

where $A=\mu C+a E_{w}$ and $B=2 a \sigma^{2} C\left[1+\tau_{w} b /(C \Delta V)\right]$.

I-O curve. The I-O curve is specified by the spike rate as a function of input strength. Here we consider two types of I-O curves: a time-varying (adapting) I-O curve and the steady-state I-O curve. In particular, we obtain the adapting I-O curve as the instantaneous spike rate response to a sustained input step (with a small baseline input) as a function of step size. This curve changes (adapts) over time, and it eventually converges to the steady-state I-O curve. As arguments of these (adapting and steady-state) I-O functions we consider presynaptic spike rates (see Figs. $2 C$ and $4 B$ and Eq. 38), input mean and standard deviation ${ }^{1}$ (see Figs. $2 D$ and $4 B$ and $E q$. 36 ), and input mean for fixed values of input standard deviation (see Fig. $8 A$ ).

${ }^{1}$ Note that because of two arguments we obtain a surface instead of a curve in this case.
ISI distribution. We calculate the ISI distribution for an aEIF neuron that has reached a steady-state spike rate $r_{\infty}:=\lim _{t \rightarrow \infty} r(t)$ by solving the so-called first passage time problem (Risken 1996; Tuckwell 1988). Consider an initial condition where the neuron has just emitted a spike and the refractory period has passed. That is, the membrane voltage is at the reset value $V_{\mathrm{r}}$ and the adaptation current, which we have replaced by its trial average (see above), takes the value $\bar{w}_{0}$, where $\bar{w}_{0}$ will be determined self-consistently (see below). In each of $N$ (simultaneous) trials, we follow the dynamics of the neuron given by $d V_{i} / d t=\left[I_{\text {ion }}\left(V_{i} ; \bar{w}\right)+I_{\text {syn }}(t)\right] / C, d \bar{w} / d t=$ $\left[a\left(1 / N \sum_{i=1}^{N} V_{i}-E_{w}\right)-\bar{w}\right] / \tau_{w}$, until its membrane voltage crosses the value $V_{\mathrm{s}}$ and record that spike time $T_{i}$. The set of times $T_{i}+T_{\text {ref }}$ then gives the ISI distribution. Finally, we determine $\bar{w}_{0}$ by imposing that the mean ISI matches with the known steady-state spike rate, i.e., $1 / N \sum_{i=1}^{N} T_{i}+T_{\text {ref }}=r_{\infty}^{-1}$. According to this calculation scheme, the ISI distribution can be obtained in the limit $N \rightarrow \infty$ by solving the Fokker-Planck system Eqs. 8 and 9 with mean adaptation current governed by

$$
\tau_{w} \frac{d \bar{w}}{d t}=a\left(\langle V\rangle_{p(V, t)}-E_{w}\right)-\bar{w},
$$

subject to the boundary conditions $(12)$ and initial conditions $p(V, 0)=$ $\delta\left(V-V_{\mathrm{r}}\right), \bar{w}(0)=\bar{w}_{0}$. Note that the reinjection condition $E q .11$ is omitted (see also the difference between Eqs. 10 and 20) because here each trial $i$ ends once $V_{i}(t)$ crosses the value $V_{\mathrm{s}}$. The ISI distribution is given by the probability flux at $V_{\mathrm{s}}$ (Tuckwell 1988; Ostojic 2011), taking into account the refractory period

$$
p_{\text {ISI }}(T)=\left\{\begin{array}{ll}
q\left(V_{\mathrm{s}}, T-T_{\text {ref }}\right) & \text { for } T \geq T_{\text {ref }} \\
0 & \text { for } T<T_{\text {ref }}
\end{array} .\right.
$$

Finally, $\bar{w}_{0}$ is determined self-consistently by requiring $\langle T\rangle_{p_{\text {ISI }}}=r_{\infty}^{-1}$. The coefficient of variation $(\mathrm{CV})$ of ISIs is then calculated as

$$
\mathrm{CV}:=\frac{\sqrt{\left\langle T^{2}\right\rangle_{p_{\text {ISI }}}-\langle T\rangle_{p_{\text {ISI }}}^{2}} .}{\langle T\rangle_{p_{\text {ISI }}}}
$$

An ISI CV value of 0 indicates regular, clock-like spiking, whereas for spike times generated by a Poisson process the ISI CV assumes a value of 1 . For a demonstration of the ISI calculation scheme described above, see Fig. 1. The results based on the Fokker-Planck equation and numerical simulations of the aEIF model with fluctuating input are presented for an increased subthreshold and spiketriggered adaptation current in separation.

ISI CV for the aPIF model. To calculate the ISI CV we need the first two ISI moments, cf. Eq. 22. The mean ISI for the aPIF neuron model is simply calculated by the inverse of the steady-state spike rate, cf. Eq. 18, derived in the previous section,

$$
\langle T\rangle_{p_{\text {ISI }}}=r_{\infty}^{-1}=\frac{\Delta V+\tau_{w} b / C}{\mu_{a}},
$$

where we consider $\mu_{a}>0$ (here and in the following). We approximate the second ISI moment by solving the first passage time problem for the Langevin equation

$$
\frac{d V}{d t}=\mu_{a}-\frac{\bar{w}_{0}}{C} \exp \left(-t / \tau_{w}\right)+\sigma \eta(t)
$$

with initial membrane voltage $V_{\mathrm{r}}$ and boundary voltage $V_{\mathrm{s}}$. That is, we replace $\langle V\rangle_{p(V, t)}$ by its steady-state value $\langle V\rangle_{\infty}$ in $E q$. 20, which is justified by large $\tau_{w}$ (as already assumed). The first passage time density (which is equivalent to $p_{\text {ISI }}$ ) and the associated first two moments for this type of Langevin equation can be calculated as power series in the limit of small $\bar{w}_{0}$ (Urdapilleta 2011). $\bar{w}_{0}$ is then determined self-consistently by imposing Eq. 23. Here we approximate the second ISI moment by using only the most dominant term of 
A

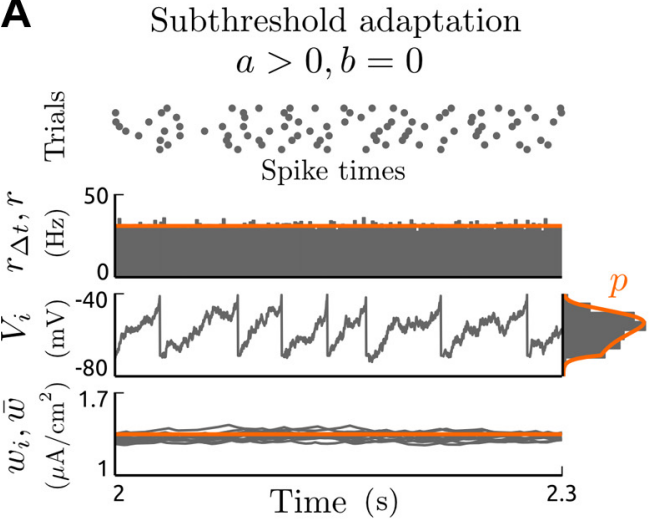

Spike-triggered adaptation $a=0, b>0$
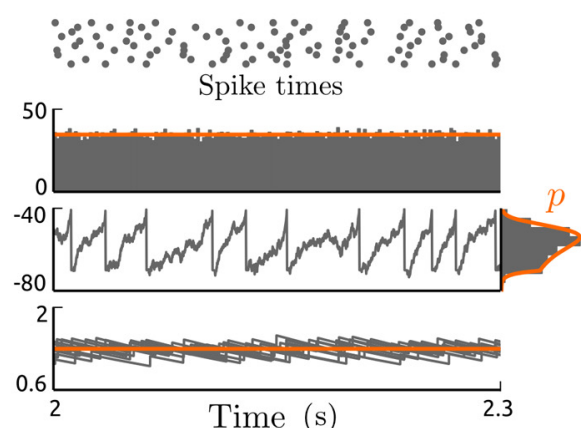

Time $(\mathrm{s})$

B
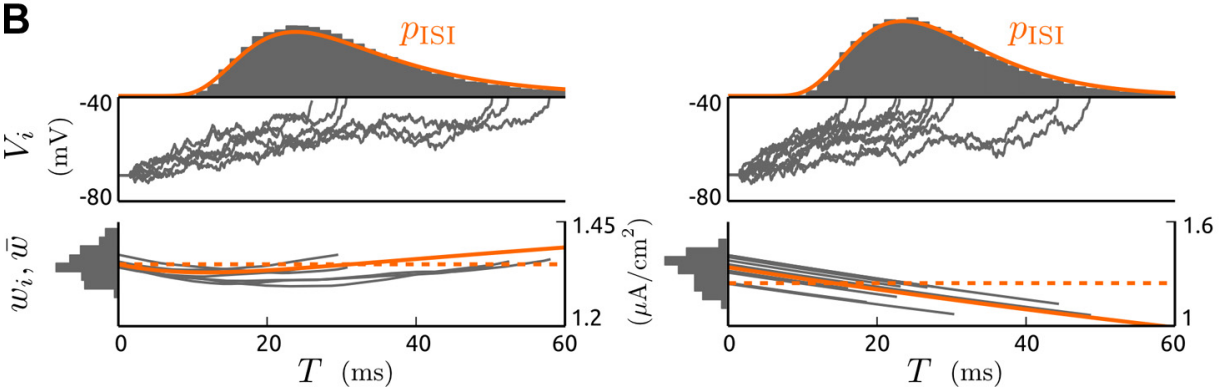

Fig. 1. Steady-state spike rates and interspike interval (ISI) distributions of single neurons. A, top to bottom: spike times, instantaneous spike rate $\left(r_{\Delta t}\right)$ histogram, membrane voltage $\left(V_{i}\right)$, membrane voltage histogram, and adaptation current $\left(w_{i}\right)$ of an (adapted) adaptive exponential integrate-and-fire (aEIF) neuron with $a=0.06 \mathrm{mS} / \mathrm{cm}^{2}, b=0$ (left), and $a=0, b=0.18 \mu \mathrm{A} / \mathrm{cm}^{2}$ (right) driven by a fluctuating input current with $\mu=2.5 \mathrm{mV} / \mathrm{ms}, \sigma=2 \mathrm{mV} / \sqrt{\mathrm{ms}}$ for $N=5,000$ trials. Spike times and adaptation current are shown for a subset of 10 trials, the membrane voltage is shown for one trial. Results from numerical simulations are shown in grey. Results obtained using the Fokker-Planck equation are indicated by orange lines and include the instantaneous spike rate $(r)$, the membrane potential distribution $(p)$, and the mean adaptation current $(\bar{w}), r, p$, And $\bar{w}$ were calculated from the Eqs. 13,8 , and 10 , respectively. These quantities have reached their steady state here. The time bin for $r_{\Delta t}$ was $\Delta t=2 \mathrm{~ms}$; for the other parameter values see MATERIALS AND METHODS. $B$, top: ISI histogram corresponding to the $N$ trials in $A$ and ISI distribution ( $p_{\text {ISI }}$, orange line) calculated via the first passage time problem $(E q .21)$. $B$, middle and bottom: membrane voltage and adaptation current trajectories from 1 trial in $A$ but rearranged such that just after each spike the time is set to zero. Histograms for the adaptation current just after the spike times are included. The time-varying mean adaptation current from the first passage time problem $(E q$. 20$)$ and the steady-state mean adaptation current from $A(E q .10)$ are indicated by solid and dashed orange lines, respectively. All histograms (in $A$ and $B$ ) represent the data from all $N$ trials.

the power series, which yields (the zeroth order approximation) (Urdapilleta 2011),

$$
\left\langle T^{2}\right\rangle_{p_{\text {ISI }}}=\frac{\sigma^{2} \Delta V+\mu_{a} \Delta V^{2}}{\mu_{a}^{3}} .
$$

Including terms of higher order leads to a complicated expression for $\left\langle T^{2}\right\rangle_{p_{\text {ISI }}}$, which has to be evaluated numerically. We additionally considered the first order term (not shown) and compared the results of both approximations (see RESULTS). Effectively, the approximation above, Eq. 25, is valid for small levels of spike-triggered adaptation current and mean input, since $\bar{w}_{0}$ increases with $b$ and $\mu$. Combining Eqs. 22, 23, and 25 the ISI CV reads

$$
\mathrm{CV}=\frac{\sqrt{\sigma^{2} \Delta V / \mu_{a}-\tau_{w}^{2} b^{2} / C^{2}-2 \tau_{w} b \Delta V / C}}{\Delta V+\tau_{w} b / C} .
$$

Neuronal network. To investigate the effects of recurrent (inhibitory) synaptic inputs on the neuronal response properties (spike rates and ISIs), we consider a network instead of a single neuron, consisting of $N_{\mathcal{E}}$ excitatory and $N_{\mathcal{I}}$ inhibitory aEIF neurons (with separate parameter sets). The two populations are recurrently coupled in the following way (see Fig. 4A). Each excitatory neuron receives inputs from $K_{c s}^{\text {ext }}$ external excitatory neurons which produce instantaneous PSPs of magnitude $J_{\varepsilon \mathcal{E}}^{\text {ext }}$ with Poisson rate $r_{\mathcal{E}}^{\text {ext }}(t)$. Analogously, each inhibitory neuron receives inputs from $K_{\mathcal{T E}}^{\text {ext }}{ }^{\mathcal{E}}$ external excitatory neurons producing instantaneous PSPs of magnitude $J_{\mathcal{T E}}^{\text {ext }}$ with Poisson rate $r_{\mathcal{I E}}^{\text {ext }}(t)$. In addition, each excitatory neuron receives inputs from
$K_{\mathcal{~} T^{\mathrm{rec}}}^{\mathrm{r}}$ randomly selected inhibitory neurons of the network with synaptic strength (i.e., instantaneous PSP magnitude) $J_{\mathcal{S T}}^{\text {rec }}$ and each inhibitory neuron receives inputs from $K_{\mathcal{T E}}^{\mathrm{rec}}$ randomly selected excitatory neurons of the network with synaptic strength $J_{\mathcal{T E}}^{\mathrm{rec}}$. This network setup was chosen to examine the effects caused by recurrent inhibition and compare them to the effects produced by external inhibition for single neurons described above. To reduce the parameter space, recurrent connections within the two populations in the network were therefore omitted. The total synaptic current for each neuron of the network can be described using $E q$. 4, where the parameters $\mu(t)$ and $\sigma(t)$ for excitatory neurons are given by

$$
\begin{gathered}
\mu(t)=J_{\mathcal{E} \mathcal{E}}^{\mathrm{ext}} K_{\mathcal{E}}^{\mathrm{ext}} r_{\mathcal{E}}^{\mathrm{ext}}(t)+J_{\mathcal{E} \mathcal{I}}^{\mathrm{rec}} K_{\mathcal{E} \mathcal{I}}^{\mathrm{rec}} r_{\mathcal{I}}^{\mathrm{pop}}(t), \\
\sigma(t)^{2}=\left(J_{\mathcal{E}}^{\mathrm{ext}}\right)^{2} K_{\mathcal{E} \mathcal{E}}^{\mathrm{ext}} r_{\mathcal{E}}^{\mathrm{ext}}(t)+\left(J_{\mathcal{E}}^{\mathrm{rec}}\right)^{2} K_{\mathcal{E} \mathcal{I}}^{\mathrm{rec}} r_{\mathcal{I}}^{\mathrm{pop}}(t)
\end{gathered}
$$

and for inhibitory neurons,

$$
\begin{gathered}
\mu(t)=J_{\mathcal{I E}}^{\mathrm{ext}} K_{\mathcal{I E}}^{\mathrm{ext}} r_{\mathcal{I E}}^{\mathrm{ext}}(t)+J_{\mathcal{I E}}^{\mathrm{rec}} K_{\mathcal{I E}}^{\mathrm{rec}} r_{\mathcal{E}}^{\mathrm{pop}}(t), \\
\sigma(t)^{2}=\left(J_{\mathcal{I}}^{\mathrm{ext}}\right)^{2} K_{\mathcal{I}}^{\mathrm{ext}} r_{\mathcal{I E}}^{\mathrm{ext}}(t)+\left(J_{\mathcal{I E}}^{\mathrm{rec}}\right)^{2} K_{\mathcal{I E}}^{\mathrm{rec}} r_{\mathcal{E}}^{\mathrm{pop}}(t)
\end{gathered}
$$

(Brunel 2000; Augustin et al. 2013). $r_{\varepsilon}^{\mathrm{pop}}(t)$ and $r_{\tau}^{\mathrm{pop}}(t)$ are the spike rates of the excitatory and inhibitory neurons of the network, respectively. Here we consider large populations of neurons instead of a large number of trials. In fact, averaging over a large number of trials in this setting is equivalent to averaging over large populations due to the random and sparse connectivity. In the limit $N_{\mathcal{E}}, N_{\mathcal{I}} \rightarrow \infty$ we obtain a system two coupled Fokker-Planck equations, one for the excitatory population, described by Eqs. 8-13, 27, and 28, and one for the 
inhibitory population, given by Eqs. 8-13, 29, and 30. Note that $r(t)$ in Eqs. 10 and 13 is replaced by the spike rates of the excitatory and inhibitory populations, $r_{\varepsilon}^{\mathrm{pop}}(t)$ and $r_{\tau}^{\mathrm{pop}}(t)$, respectively. We solve this system to obtain the steady-state spike rate for each population, $r_{\text {pop }}^{\text {pop }}$ and $r_{\tau \infty}^{\text {pop }}$. Once these quantities are known, we calculate the ISI distribution, cf. Eq. 21, for the excitatory population (i.e., for any neuron of that population) as described above, using Eqs. 27 and 28 for the (steady-state) moments of the synaptic current. The neuron model parameter values were as above for the single neuron, with $a=$ $0.015 \mathrm{mS} / \mathrm{cm}^{2}, b=0.1 \mu \mathrm{A} / \mathrm{cm}^{2}$ for excitatory neurons and $a=b=$ 0 for inhibitory neurons, since adaptation was found to be weak in fast-spiking interneurons compared with pyramidal neurons (La Camera et al. 2006). The network parameter values were $J_{\mathcal{E \varepsilon}}^{\text {ext }}=J_{\mathcal{T E}}^{\text {ext }}=0.15$ $\mathrm{mV}, K_{\mathcal{E S}}^{\mathrm{ext}}=K_{\mathcal{T S}}^{\text {ext }}=800$, constant $r_{\mathcal{E S}}^{\text {ext }} \in[0,80] \mathrm{Hz}, J_{\mathcal{E}}^{\text {rec }} \in^{\mathcal{I E}}[-0.75$,

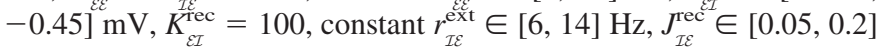
$\mathrm{mV}$, and $K_{\mathcal{T S}}^{\mathrm{rec}}=400$.

Numerical solution. We treated the Fokker-Planck equations for the aPIF model analytically. In case of the aEIF model, we solved these equations forward in time using a first-order finite volume method on a nonuniform grid with 512 grid points in the interval $\left[-200 \mathrm{mV}, V_{\mathrm{s}}\right]$ and the implicit Euler integration method with a time step of $0.1 \mathrm{~ms}$ for the temporal domain. For more details on the numerical solution, we refer to Augustin et al. (2013).

Detailed conductance-based neuron model. For validation purposes we used a biophysical Hodgkin-Huxley-type neuron model with different types of slow $\mathrm{K}^{+}$current. The membrane voltage $V$ of this neuron model obeys the current balance equation

$$
C \frac{d V}{d t}=I-I_{\mathrm{L}}-I_{\mathrm{Na}}-I_{\mathrm{K}}-I_{\mathrm{Ca}}-I_{\mathrm{Ks}},
$$

where $C=1 \mu \mathrm{F} / \mathrm{cm}^{2}$ is the membrane capacitance and $I$ denotes the injected current. The ionic currents consist of a leak current, $I_{\mathrm{L}}=$ $g_{\mathrm{L}}\left(V-E_{\mathrm{L}}\right)$, a spike-generating $\mathrm{Na}^{+}$current, $I_{\mathrm{Na}}=g_{\mathrm{Na}}(V)\left(V-E_{\mathrm{Na}}\right)$, a delayed rectifier $\mathrm{K}^{+}$current, $I_{\mathrm{K}}=g_{\mathrm{K}}(V)\left(V-E_{\mathrm{K}}\right)$, a high-threshold $\mathrm{Ca}^{2+}$ current, $I_{\mathrm{Ca}}=g_{\mathrm{Ca}}(V)\left(V-E_{\mathrm{Ca}}\right)$, and a slow $\mathrm{K}^{+}$current $I_{\mathrm{Ks}} \cdot g_{\mathrm{x}}$ Denote the conductances of the respective ion channels and $E_{\mathrm{x}}$ are the reversal potentials. We separately considered three types of slow $\mathrm{K}^{+}$ current: a $\mathrm{Ca}^{2+}$-activated current $\left(I_{\mathrm{Ks}} \equiv I_{\mathrm{KCa}}\right)$ which is associated with the slow after-hyperpolarization following a burst of spikes (Brown and Griffith 1983), a $\mathrm{Na}^{+}$-activated current $\left(I_{\mathrm{Ks}} \equiv I_{\mathrm{KNa}}\right)$ (Schwindt et al. 1989), and the voltage-dependent muscarine-sensitive (M type) current $\left(I_{\mathrm{Ks}} \equiv I_{\mathrm{M}}\right)$ (Brown and Adams 1980). The leak current depends linearly on the membrane potential. All other ionic currents depend on $V$ in a nonlinear way as described by the HodgkinHuxley formalism. We adopted the somatic model from (Wang et al. 2003) and included the M current with dynamics described (for the soma) by (Mainen and Sejnowski 1996). The conductances underlying the currents $I_{\mathrm{Na}}, I_{\mathrm{K}}, I_{\mathrm{Ca}}$, and $I_{\mathrm{M}}$ are given by $g_{\mathrm{Na}}=\bar{g}_{\mathrm{Na}} m_{\infty}^{3} h$, $g_{\mathrm{K}}=\bar{g}_{\mathrm{K}} n^{4}, g_{\mathrm{Ca}}=\bar{g}_{\mathrm{Ca}} s_{\infty}^{2}$ and $g_{\mathrm{M}}=\bar{g}_{\mathrm{M}} u$, respectively, with steady-state gating variables $m_{\infty}=\alpha_{m} /\left(\alpha_{m}+\beta_{m}\right), \alpha_{m}=-0.4(V+33) /\{\exp [-(V+$ $33) / 10]-1\}, \beta_{m}=16 \exp [-(V+58) / 12]$, and $s_{\infty}=1 /\{1+\exp [-(V+$ 20)/9]\}. The dynamic gating variables $x \in\{h, n, u\}$ are governed by

$$
\frac{d x}{d t}=\alpha_{x}(1-x)-\beta_{x} x
$$

where $\alpha_{h}=0.28 \exp [-(V+50) / 10], \beta_{h}=4 /\{1+\exp [-(V+$ $20) / 10]\}, \alpha_{n}=-0.04(V+34) /\{\exp [-(V+34) / 10]-1\}, \beta_{n}=0.5$ $\exp [-(V+44) / 25], \alpha_{u}=3.209 \cdot 10^{-4}(V+30) /\{1-\exp [-(V+$ $30) / 9]\}$ and $\beta_{u}=-3.209 \cdot 10^{-4}(V+30) /\{1-\exp [(V+30) / 9]\}$. The channel opening and closing rates $\alpha_{x}$ and $\beta_{x}$ are specified in $\mathrm{ms}^{-1}$ and the membrane voltage $V$ in the equations above is replaced by its value in $\mathrm{mV}$. The conductance for the $\mathrm{Ca}^{2+}$-activated slow $\mathrm{K}^{+}$ current $I_{\mathrm{KCa}}$ is given by $g_{\mathrm{KCa}}=\bar{g}_{\mathrm{KCa}}[\mathrm{Ca}] /([\mathrm{Ca}]+\kappa)$, where the intracellular $\mathrm{Ca}^{2+}$ concentration $[\mathrm{Ca}]$ satisfies

$$
\frac{d[\mathrm{Ca}]}{d t}=-\alpha_{\mathrm{Ca}} I_{\mathrm{Ca}}-\frac{[\mathrm{Ca}]}{\tau_{\mathrm{Ca}}}
$$

with $\alpha_{\mathrm{Ca}}=6.67 \cdot 10^{-4} \mu \mathrm{M} \cdot \mathrm{cm}^{2} /(\mu \mathrm{A} \cdot \mathrm{ms}), \tau_{\mathrm{Ca}}=240 \mathrm{~ms}$, and $\kappa=0.03$ $\mathrm{mM}$. The conductance for the $\mathrm{Na}^{+}$-activated slow $\mathrm{K}^{+}$current $I_{\mathrm{KNa}}$ is described by $g_{\mathrm{KNa}}=\bar{g}_{\mathrm{KNa}} 0.37 /\left\{1+(\varrho /[\mathrm{Na}])^{3.5}\right\}$, where $\varrho=38.7$ $\mathrm{mM}$ and the intracellular $\mathrm{Na}^{+}$concentration $[\mathrm{Na}]$ is governed by

$$
\frac{d[\mathrm{Na}]}{d t}=-\alpha_{\mathrm{Na}}-3 \varphi\left(\frac{[\mathrm{Na}]^{3}}{[\mathrm{Na}]^{3}+\vartheta^{3}}-\gamma\right)
$$

with $\alpha_{\mathrm{Na}}=0.3 \mu \mathrm{M} \cdot \mathrm{cm}^{2} /(\mu \mathrm{A} \cdot \mathrm{ms}), \varphi=0.6 \mu \mathrm{M} / \mathrm{ms}, \vartheta=15 \mathrm{mM}$, and $\gamma=0.132$. We varied the peak conductances of the three slow $\mathrm{K}^{+}$ currents $I_{\mathrm{KCa}}, I_{\mathrm{KNa}}, I_{\mathrm{M}}$ in the ranges $\bar{g}_{\mathrm{KCa}} \in[2,8] \mathrm{mS} / \mathrm{cm}^{2}, \bar{g}_{\mathrm{KNa}} \in$ $[2,8] \mathrm{mS} / \mathrm{cm}^{2}$ (Wang et al. 2003), and $\bar{g}_{\mathrm{M}} \in[0.1,0.4] \mathrm{mS} / \mathrm{cm}^{2}$ (Mainen and Sejnowski 1996). The remaining parameter values were $C=1 \mu \mathrm{F} / \mathrm{cm}^{2}, g_{\mathrm{L}}=0.1 \mathrm{mS} / \mathrm{cm}^{2}, E_{\mathrm{L}}=-65 \mathrm{mV}, E_{\mathrm{Na}}=55 \mathrm{mV}$, $E_{\mathrm{K}}=-80 \mathrm{mV}$, and $E_{\mathrm{Ca}}=120 \mathrm{mV}$ (Wang et al. 2003).

The differences of the slow $\mathrm{K}^{+}$currents $\left(I_{\mathrm{KCa}}, I_{\mathrm{KNa}}\right.$, and $\left.I_{\mathrm{M}}\right)$ is effectively expressed by their steady-state voltage dependence and time constants. Therefore, we further considered a range of biologically plausible steady-state conductance-voltage relationships and timescales using the generic description of a slow $\mathrm{K}^{+}$current, $I_{\mathrm{Ks}}=$ $\bar{g}_{\mathrm{Ks}} \omega(V)\left(V-E_{\mathrm{K}}\right)$, with peak conductance $\bar{g}_{\mathrm{Ks}}$ and gating variable $\omega(V)$ given by

$$
\tau_{\omega} \frac{d \omega}{d t}=\omega_{\infty}(V)-\omega
$$

where $\omega_{\infty}(V)=1 /\{1+\exp [-(V-\alpha) / \beta]\}$. The shape of the steady-state curve $\omega_{\infty}(V)$ was changed by the parameters $\alpha \in[-40$, $-10] \mathrm{mV}$ (half-activation voltage), $\beta \in[6,12] \mathrm{mV}$ (inverse steepness), and the time constant $\tau_{\omega}$ was varied in $[100,300] \mathrm{ms}$. The model equations were solved using a second order Runge-Kutta integration method with a time step of $10 \mu \mathrm{s}$.

To examine the effects of slow $\mathrm{K}^{+}$currents on the I-O curve and ISI variability for noisy input, we additionally considered the synaptic current described by Eq. 4 for the detailed neuron model, i.e., we used $I \equiv I_{\text {syn }}$ in $E q .31$.

Subthreshold and spike-triggered components of biophysical slow $K^{+}$currents. To assess how the relative levels of subthreshold adaptation conductance (parameter $a$ ) and spike-triggered adaptation current increments (parameter $b$ ) in the aEIF model reflect different types of slow $\mathrm{K}^{+}$current, we quantified their subthreshold and spike-triggered components using the detailed conductance-based neuron model. First, we fit the steady-state adaptation current $w_{\infty}=$ $a\left(V-E_{w}\right)$ from the aEIF model to the respective $\mathrm{K}^{+}$current $I_{\mathrm{Ks}}$ of the Hodgkin-Huxley-type model in steady-state over a range of subthreshold values for the membrane voltage, $V \in[-70,-60] \mathrm{mV}$. Thereby we obtained an estimate for $a$. In the second step, we measured the absolute and relative change of $I_{\mathrm{Ks}}$ elicited by one spike. This was done by injecting a slowly increasing current ramp into the detailed model neuron and measuring $I_{\mathrm{Ks}}$ just before and after the first spike that occurred. Specifically, the absolute change of current caused by a spike was given by $\Delta I_{\mathrm{Ks}}:=I_{\mathrm{Ks}}\left(t_{\mathrm{s}}^{\text {post }}\right)-I_{\mathrm{Ks}}\left(t_{\mathrm{s}}^{\text {pre }}\right)$, where the time points $t_{\mathrm{s}}^{\mathrm{pre}}$ and $t_{\mathrm{s}}^{\mathrm{post}}$ were defined by the times at which the membrane potential crosses a value close to threshold (we chose -50 $\mathrm{mV}$ ) during the upswing and downswing of the spike, respectively. $\Delta I_{\mathrm{Ks}}$ provides an estimate for $b$. The relative change of $\mathrm{K}^{+}$current was $\Delta I_{\mathrm{Ks}}^{\mathrm{rel}}:=\Delta I_{\mathrm{Ks}} / I_{\mathrm{Ks}}\left(t_{\mathrm{s}}^{\mathrm{pre}}\right)$. Here we only fitted the parameters $a$ and $b$ of the aEIF model. For an alternative fitting procedure which comprises all model parameters, we refer to (Brette and Gerstner 2005).

\section{RESULTS}

Spike rate adaptation, gain, and threshold modulation in single neurons. We first examine the responses of single aEIF neurons with and without an adaptation current, receiving 
inputs from stochastically spiking presynaptic excitatory and inhibitory neurons. The compound effect of the individual synaptic inputs is represented by an ongoing fluctuating input current whose mean and standard deviation depend on the synaptic strengths and spike rates of the presynaptic cells (cf. Eqs. 4-6 in MATERIALS AND METHODS and Fig. 2A). The neurons naturally respond to a sudden increase in spike rate of the presynaptic neurons (an input step) with an abrupt increase in spike rate and mean membrane voltage (see Fig. $2 B$ ). Without an adaptation current, both quantities remain unchanged after that increase. In case of a purely subthreshold adaptation current ( $a>0, b=0$ in the aEIF model), which is present already in absence of spiking, the rapid increase of mean membrane voltage causes the mean adaptation current to build up slowly, which in turn leads to a gradual decrease in spike rate and mean membrane voltage. Note that the mean membrane voltage is decreased in the absence of spiking (before the increase of input) compared with the neuron without adapta- tion. In case of a purely spike-triggered adaptation current ( $a=$ $0, b>0$ in the aEIF model), the sudden increase in spike rate leads to an increase of mean adaptation current, which again causes the spike rate and mean membrane voltage to decrease gradually.

The adapting I-O curve of neurons with and without an adaptation current, that is, the time-varying spike rate response to a step in presynaptic spike rates as a function of the step size, is shown in Fig. 2C. Interestingly, the two types of adaptation current affect the spike rate response in different ways. A subthreshold adaptation current shifts the I-O curve subtractively and thus increases the threshold for spiking. In addition, it decreases the response gain for low (output) spike rates. If the adaptation current is driven by spikes on the other hand, the I-O curve changes divisively, that is, the response gain is reduced over the whole range of spike rate values but the response threshold remains unchanged. It can be recognized that for a given type of adaptation current the adapting I-O
A

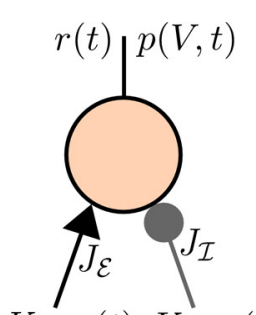

$K_{\mathcal{E}} r_{\mathcal{E}}(t) K_{\mathcal{I}} r_{\mathcal{I}}(t)$

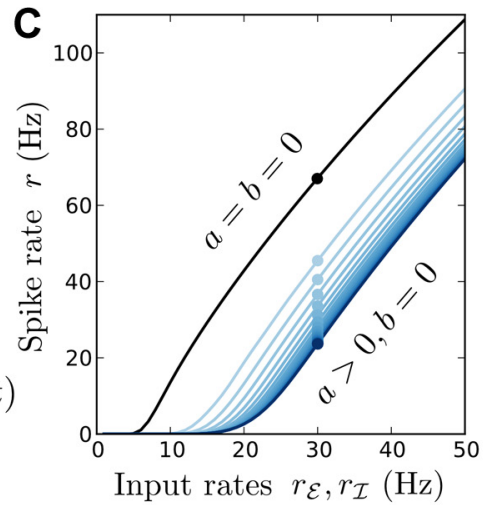

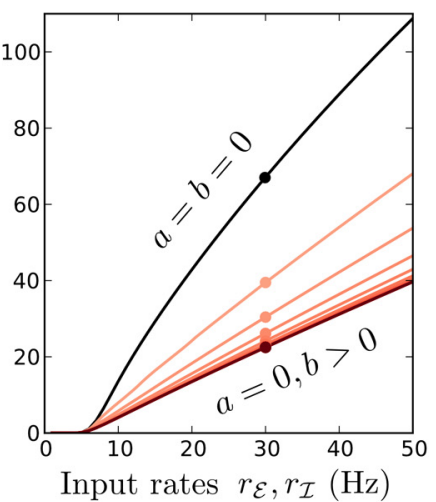

Input rates $r_{\mathcal{E}}, r_{\mathcal{I}}(\mathrm{Hz})$
B
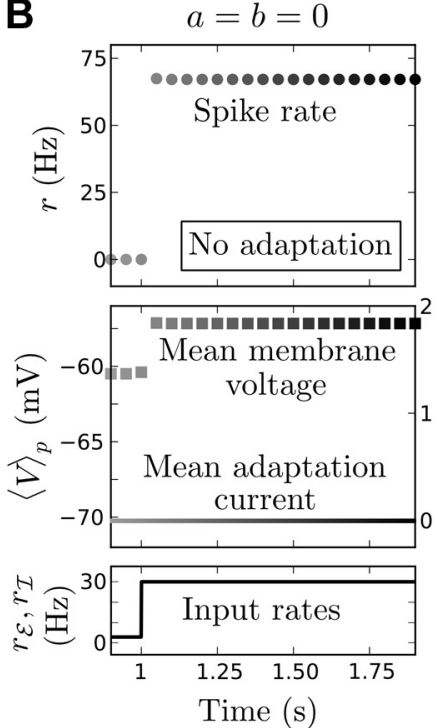
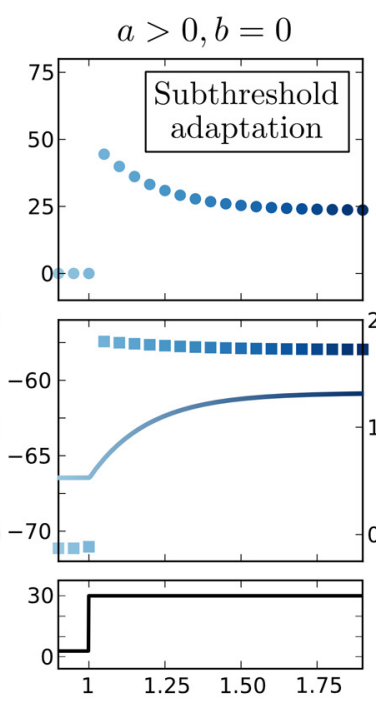

Time (s)
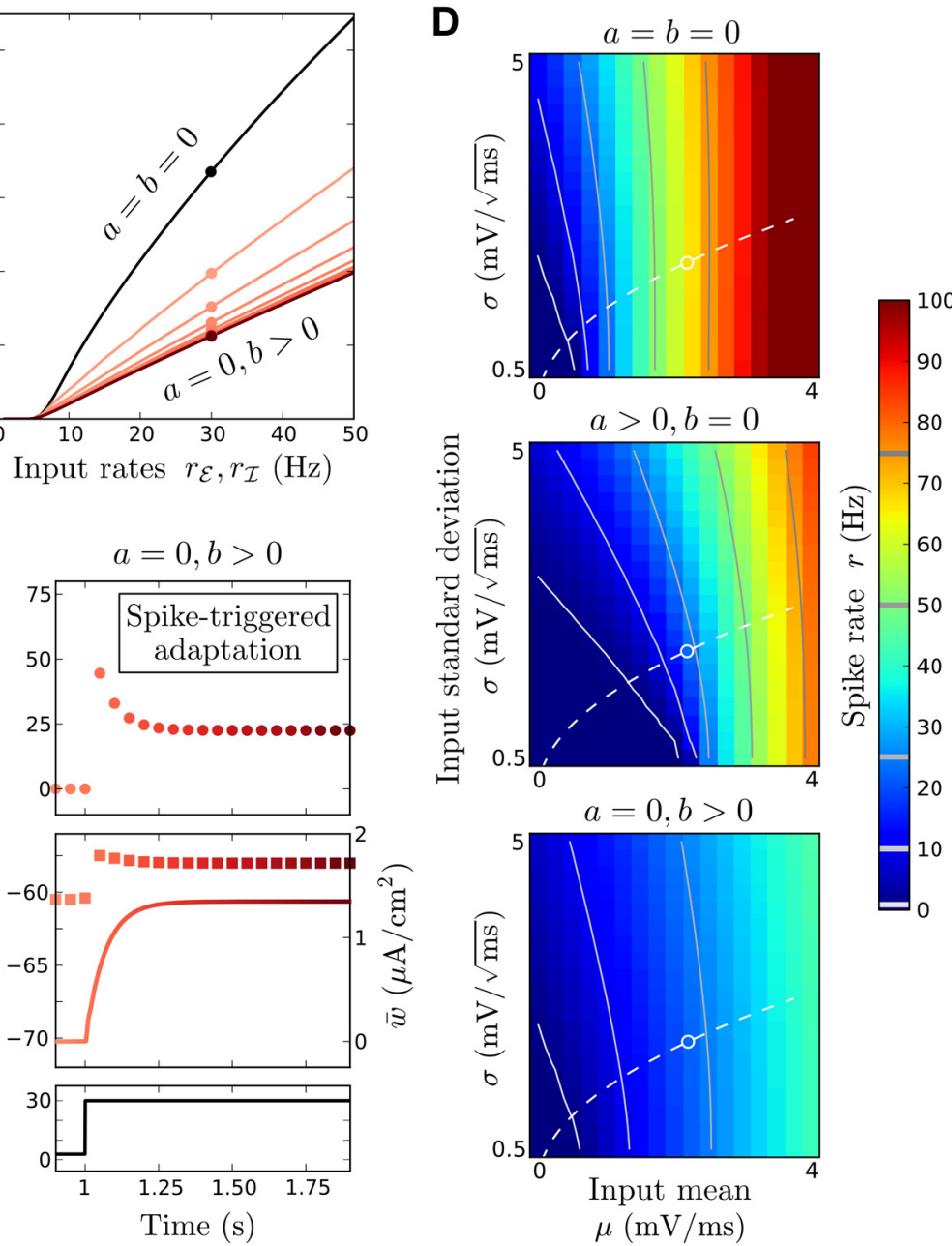

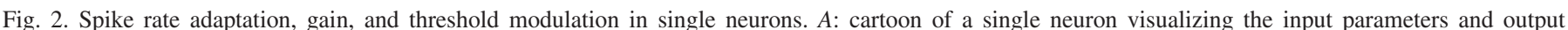

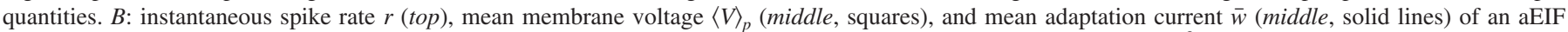

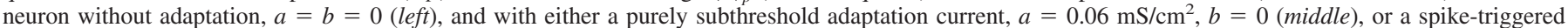

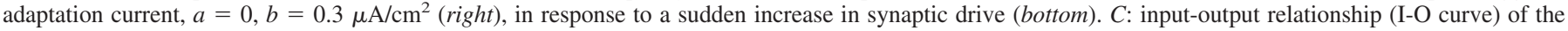

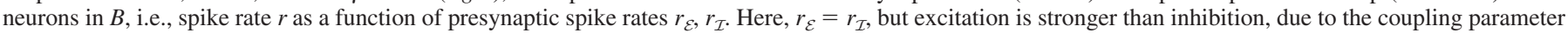

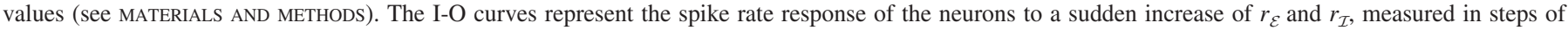

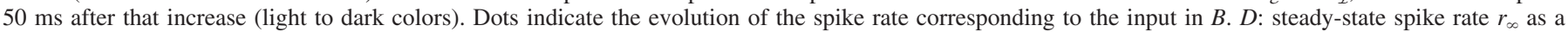

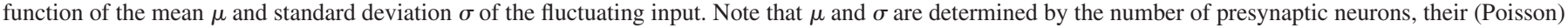

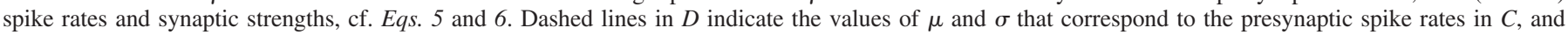
circles mark the values of the moments corresponding to the increased input in $B$. 
curve evaluated shortly after the input steps and the steadystate I-O curve are changed qualitatively in the same way. Thus, for the following parameter exploration and analytical derivation, we focus on (changes of) the steady-state I-O relationship.

We next explore the effects of an adaptation current on the steady-state spike rate for a wide range of input statistics, that is, different values of the mean $\mu$ and the standard deviation $\sigma$ of the fluctuating total synaptic input (see Fig. 2D). If excitatory and inhibitory inputs are approximately balanced, the standard deviation $\sigma$ of the compound input is large compared with its mean $\mu$. The spike rate increases with an increase of either $\mu$ or $\sigma$ or both. A subthreshold adaptation current increases the threshold for spiking in terms of $\mu$ as well as $\sigma$. A spike-triggered adaptation current, however, does not change the threshold for spiking but reduces the gain of the spike rate as a function of $\mu$ or $\sigma$. Thus the differential effects of both types of adaptation current are robust across different input configurations. Note that the I-O curve as a function of mean input $\mu$ changes additively for increased levels of standard deviation $\sigma$ while its slope (i.e., gain) decreases, particularly for small values of $\mu$. This can be recognized by the contour lines in Fig. 2D and is most prominent for increased subthreshold adaptation. Consequently, this type of adaptation current increases the sensitivity of the steady-state spike rate to noise intensity for low spike rates.

To analytically demonstrate the differential effects of subthreshold and spike-triggered adaptation currents on the (steady-state) I-O curve, we consider the aPIF neuron model, which is obtained by neglecting the leak conductance $\left(g_{\mathrm{L}}=0\right)$ in the aEIF model. This allows to derive an explicit expression for the steady-state spike rate,

$$
r_{\infty}=\frac{\mu-a\left(\langle V\rangle_{\infty}-E_{w}\right) / C}{\Delta V+\tau_{w} b / C},
$$

where the mean membrane voltage $\langle V\rangle_{\infty}$ with respect to the steady-state distribution $p_{\infty}(V)$ is given by $E q .19$ and $\Delta V:=$ $V_{\mathrm{s}}-V_{\mathrm{r}}$ is the difference between spike and reset voltage; $r_{\infty}=$ 0 for $\mu<a\left(\langle V\rangle_{\infty}-E_{w}\right) / C$ (see MATERIALS AND METHODS). Equation 36 mathematically demonstrates the subtractive component of the effect a subthreshold adaptation current $(a>0)$ produces when the mean membrane voltage is larger than the reversal potential $E_{w}$ of the $\left(\mathrm{K}^{+}\right)$adaptation current. Taking the derivative of $E q$. 36 with respect to $\mu$ further reveals that an increase of $a$ reduces the gain when the input fluctuations $(\sigma)$ are large compared with the mean $(\mu)$. A spike-triggered adaptation current $(b>0)$, however, produces a purely divisive effect that can be pronounced even for small current increments $b$ if the adaptation timescale $\tau_{w}$ is large.

Differential effects of adaptation currents on spiking variability. We next investigate how adaptation currents affect ISIs for different input statistics. For that reason we calculate the distribution of times at which the membrane voltage of an aEIF neuron crosses the threshold $V_{\mathrm{s}}$ for the first time, which is equivalent to the distribution of ISIs (see MATERIALS AND METHODS). These ISI distributions are shown in Fig. $3 A$ for neurons with different levels of subthreshold or spike-triggered adaptation and a given input. An increase of either type of adaptation current (via parameters $a$ and $b$ ) naturally increases the mean ISI. Interestingly, while subthreshold adaptation leads to
A
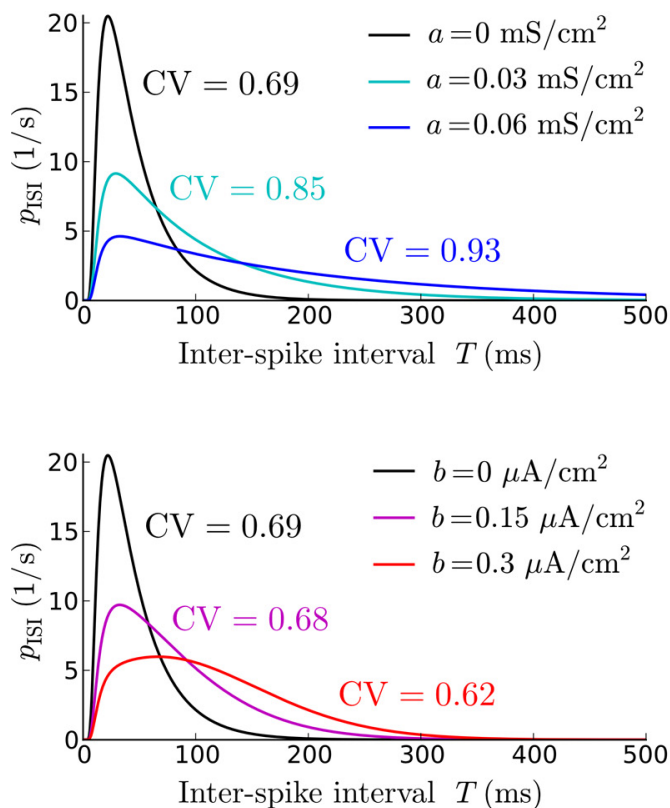

B No adaptation $a=b=0$

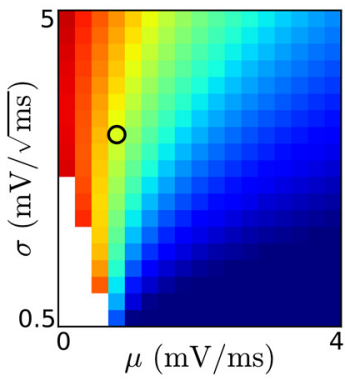

C
Subthreshold adaptation $a>0, b=0$

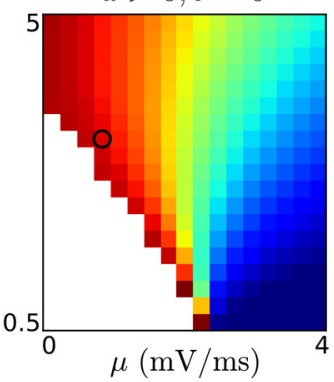

Spike-triggered adaptation $a=0, b>0$
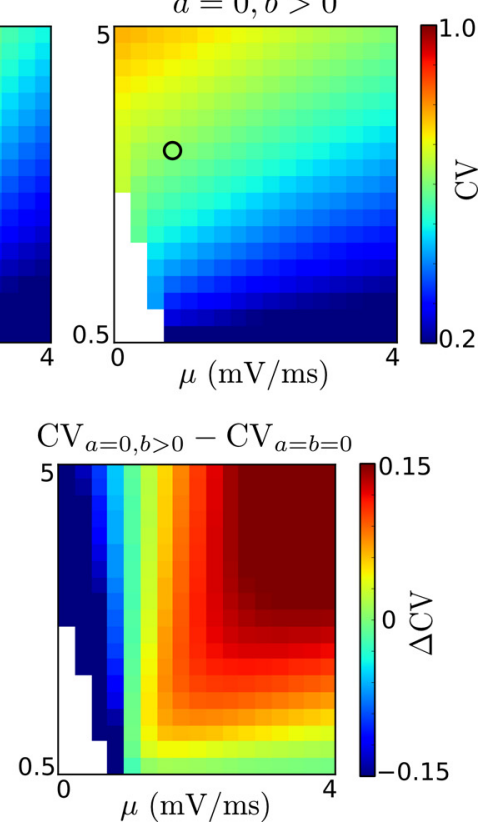
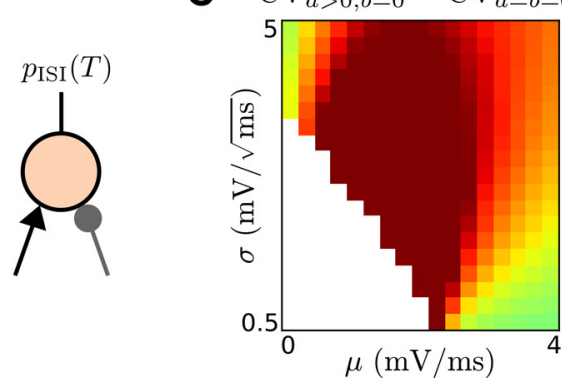

Fig. 3. Changes of spiking variability in single neurons. A: ISI distribution $\left(p_{\text {ISI }}\right)$ of a single aEIF neuron in response to a fluctuating input with mean $\mu=0.75$ $\mathrm{mV} / \mathrm{ms}$ and standard deviation $\sigma=3.25 \mathrm{mV} / \sqrt{\mathrm{ms}}$, for $a=0,0.03,0.06 \mathrm{mS} / \mathrm{cm}^{2}, b=0$ (top) and $a=0, b=0,0.15$, and $0.3 \mu \mathrm{A} / \mathrm{cm}^{2}$ (bottom). $B$ : ISI coefficient of variation (CV) as a function of $\mu$ and $\sigma$, for a neuron without adaptation, $a=b=0$ (left), and with either a subthreshold adaptation current, $a=0.06 \mathrm{mS} / \mathrm{cm}^{2}$, $b=0$ (middle), or a spike-triggered adaptation current, $a=0, b=0.3 \mu \mathrm{A} / \mathrm{cm}^{2}$ (right). Circles indicate the values of $\mu$ and $\sigma$ used in $A$. C: change of ISI CV caused by a subthreshold (left) or spike-triggered (right) adaptation current as a function of $\mu$ and $\sigma$. White regions in $B$ and $C$ indicate the parameter values for which the ISI CV was not computed, because $r_{\infty}<1 \mathrm{~Hz}$. 
ISI distributions with long tails, spike-triggered adaptation causes ISI distributions with bulky shapes. These differential effects on the shape of the ISI distribution lead to opposite changes of the CV (cf. Eq. 22), which quantifies the variability of ISIs. An increase of subthreshold adaptation current produces an increase of $\mathrm{CV}$, whereas an increase of spike-triggered adaptation current leads to a decreased ISI variability. How these effects on the CV of ISIs depend on the statistics ( $\mu$ and $\sigma$ ) of the fluctuating input is shown in Fig. 3, $B$ and $C$. With or without an adaptation current, if the mean $\mu$ is large, that is, far above threshold, and the standard deviation $\sigma$ is comparatively small, the neuronal dynamics is close to deterministic and the firing is almost periodic; hence, the CV is small. In contrast, if $\mu$ is close to the threshold and $\sigma$ is large (enough), the ISI distribution will be broad as indicated by the large CV. A subthreshold adaptation current either leads to an increased CV or leaves the ISI variability unchanged. In case of a spike-triggered adaptation current the effect on the CV depends on the input statistics. This type of adaptation current causes a decrease of the high ISI variability in the region (of the $\mu, \sigma$-plane) where the mean input $\mu$ is small and an increase of the low ISI variability for larger values of $\mu$.

We analytically derived an approximation of the ISI CV for the aPIF model, which emphasizes the opposite effects of the two types of adaptation current. It is obtained as

$$
\mathrm{CV}=\frac{\sqrt{\sigma^{2} \Delta V / \mu_{a}-\tau_{w}^{2} b^{2} / C^{2}-2 \tau_{w} b \Delta V / C}}{\Delta V+\tau_{w} b / C}
$$

(same as $E q .26$ ), where $\mu_{a}:=\mu-a\left[\langle V\rangle_{\infty}-E_{w}\right] / C$ is the effective mean input which is again assumed to be positive and takes into account the counteracting subthreshold adaptation current. The steady-state mean membrane voltage $\langle V\rangle_{\infty}$ is given by $E q .19$ (see MATERIALS AND METHODS). Equation 37 mathe- matically demonstrates that an increase of subthreshold adaptation curent $(a>0)$ causes an increase of $\mathrm{CV}$ as long as $\langle V\rangle_{\infty}$ is larger than $E_{w}$, that is, the mean membrane voltage is not too hyperpolarized. An increase of spike-triggered adaptation current $(b>0)$ on the other hand leads to a reduction of ISI variability. Note that this approximation is only valid for small values of mean input $(\mu)$ and adaptation current increment $(b)$. It does not account for the increase of $\mathrm{CV}$ caused by spiketriggered adaptation for large levels of $\mu$ (cf. Fig. 3C). Both (input dependent) effects of spike-triggered adaptation on the ISI variability can be captured by a refined approximation of the CV compared with Eq. 37 (not shown, see MATERIALS AND METHODS for an outline), which requires numerical evaluation.

Differential effects of synaptic inhibition on I-O curves. Here we examine how synaptic input received from a population of inhibitory neurons affect gain and threshold of spiking. We consider that the neuron we monitor belongs to a population of excitatory neurons which are recurrently coupled to neurons from an inhibitory population, as depicted in Fig. 4A: Each neuron of the network receives excitatory synaptic input from external neurons and additional synaptic input from a number of neurons of the other population. The specific choice of the monitored excitatory neuron does not matter because of identical model parameters within each population and sparse random connectivity (see MATERIALS AND METHODS). Figure $4 B$ shows how the steady-state I-O curve of excitatory neurons, i.e., the spike rate $r_{s-\mathrm{pop}}^{\mathrm{pa}}$ a function of the external (input) spike rate $r_{s c}^{\text {ext }}$, is changed by external excitation to the inhibitory neurons (via $r_{\tau c}^{\text {ext }}$ ) and by the strengths of the recurrent excitatory and inhibitory synapses $\left(J_{\mathcal{T E}}^{\mathrm{rec}}\right.$ and $\left.J_{\varepsilon \mathcal{T}}^{\mathrm{rec}}\right)$, respectively. An increase of external excitation to the inhibitory population (via $J_{\tau c}^{\text {ext }}$ ) changes the I-O curve subtractively, thus increasing the response threshold, while an increase of recurrent excitation to

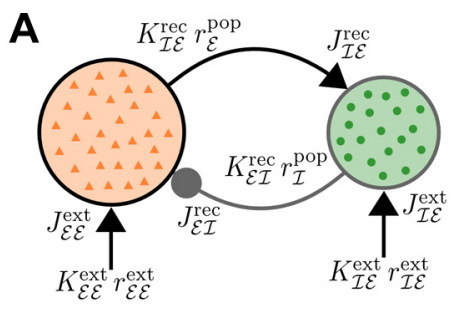

Fig. 4. Gain and threshold modulation caused by network interaction. $A$ : cartoon of the network visualizing the coupling parameters. $B$, top: steady-state spike rate of excitatory aEIF neurons, $r_{\varepsilon \infty}^{\text {pop }}$ (solid lines) and inhibitory aEIF neurons, $r_{\tau_{\infty}}^{\mathrm{pop}}$ (dashed lines), as a function of $r_{s \mathcal{s}}^{\mathrm{ext}}$, for $r_{\mathcal{T S}}^{\mathcal{E}, \mathrm{ext}}=6,10$, and $14 \mathrm{~Hz}(l e f t) ; J_{\mathcal{T E}}^{\mathrm{rec}}=0.05,0.1$, and $0.2 \mathrm{mV}$ (middle); and $J_{\mathcal{E T}}^{\mathrm{rec}}=-0.45,-0.6$, and -0.75 $\mathrm{mV}$ (right). Insets: cartoons visualizing the varied parameters as specified on the top left. If not indicated otherwise, $J^{\mathrm{rec}}=-0.6 \mathrm{mV}$, $r_{\mathcal{T E}}^{\mathrm{ext}}=10 \mathrm{~Hz}$, and $J_{\mathcal{T E}}^{\mathrm{rec}}=0.1 \mathrm{mV}$. For the other parameter values see MATERIALS AND METHODS. $B$, bottom: steady-state spike rate $r_{\mathcal{E}_{\infty}}^{\mathrm{pop}}$ as a function of the input parameters $\mu$ and $\sigma$ for the excitatory neurons. Solid lines and dots at top correspond to those of equal color at bottom.

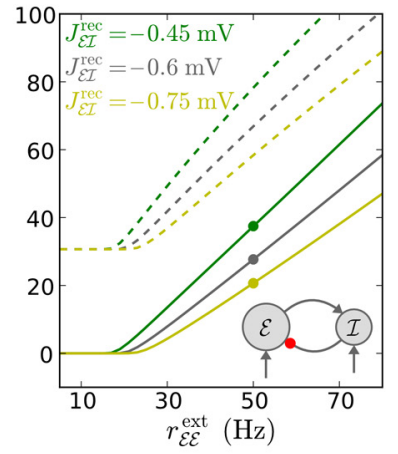

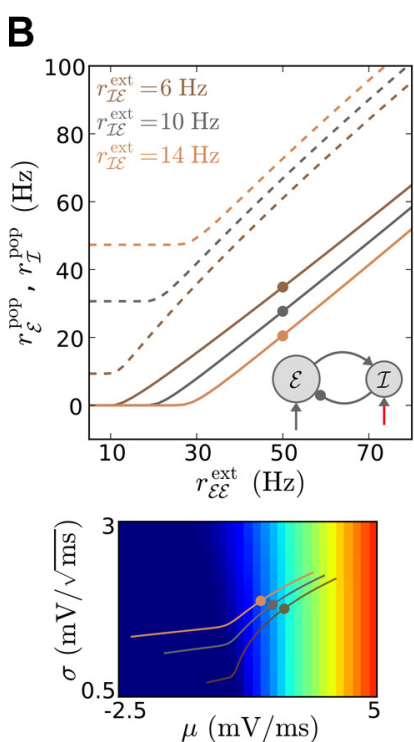
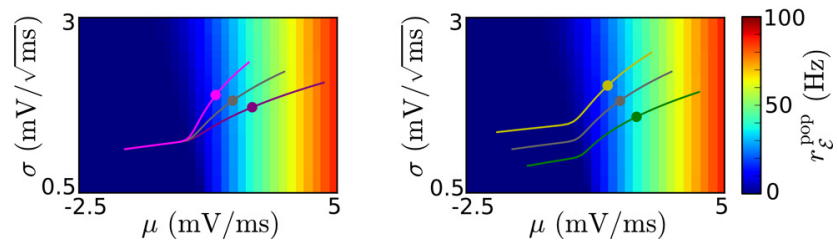
the inhibitory neurons (via $J_{\tau c}^{\text {rec}}$ ) has a purely divisive effect, that is, the gain is reduced. On the other hand, an increase of recurrent inhibition to the excitatory neurons (via $J_{s \tau}^{\text {rec }}$ ) affects the I-O curve in both ways.

We demonstrate these effects analytically for a network of perfect integrate-and-fire (PIF) model neurons (instead of aEIF neurons). That is, we disregard the adaptation current here for simplicity $(a=b=0)$, since it does not change the results qualitatively. An explicit expression for the steady-state spike rate of the excitatory neurons, $r_{\varepsilon_{\infty}}^{\mathrm{pop}}$, can be derived using Eq. 36 for all the neurons in the network with mean input $\mu$ given by $E q .27$ for excitatory neurons and by Eq. 29 for inhibitory neurons. We solve for $r_{\varepsilon_{\infty}}^{\mathrm{pop}}$ self-consistently to obtain,

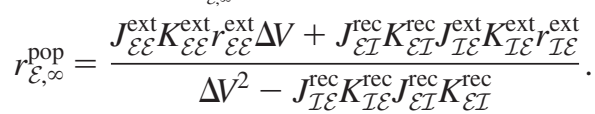

The equation above states that $r_{\varepsilon_{\infty}}^{\mathrm{pop}}$ is directly proportional to the strength of external excitation to the excitatory population, negatively proportional to the strength of external excitation to the inhibitory population (since $J_{\varepsilon T}^{\text {rec }}<0$ ) and inversely proportional to the strength of recurrent excitation, where all proportionalities include an offset. Equation 38 clearly shows that the effect of external excitation to the inhibitory population is purely subtractive (since $J_{\varepsilon T}^{\text {rec }}<0$ ), the effect of recurrent excitation (to the inhibitory population) is purely divisive, and the effect of recurrent inhibition (to the excitatory population) includes both components. For comparison, consider a single (nonadapting) PIF neuron receiving (external) excitatory and inhibitory input. With the use of Eq. 36 with mean input $\mu$ given by Eq. 5, the steady-state spike rate of this neuron reads $r_{\infty}=\left(J_{\mathcal{E}} K_{\mathcal{E}} r_{\mathcal{E}}+\right.$ $\left.J_{\mathcal{I}} K_{\mathcal{I}} r_{\mathcal{I}}\right) / \Delta V$. Thus, an increase of external inhibition affects the I-O curve of an excitatory neuron in the same way (subtractively) as an increase of external excitation to the inhibitory population within a recurrent network as described above.
Effects of synaptic inhibition on spiking variability. We next investigate how inhibitory synaptic input changes the ISI variability of the neurons (from the excitatory population) in the network described above. An increase of external excitation to the inhibitory neurons (via $r_{\tau \mathcal{T}}^{\mathrm{ext}}$ ), and the strengths of the recurrent synapses $\left(J_{\tau_{c}}^{\mathrm{rec}}\right.$ and $\left.J_{c_{\tau}}^{\mathrm{rec}}\right)$ individually, leads to an increase of the mean ISI and an increased tail of the ISI distribution, as shown in Fig. 5A. Furthermore, an increase of $r_{\mathcal{L}}^{\mathrm{ext}}$ or the magnitude of $J_{\tau \mathcal{T S}}^{\mathrm{rec}}$ or $J_{\varepsilon \tau}^{\mathrm{rec}}$, each causes the coefficient of variation of ISIs $\left(\mathrm{CV}^{\mathrm{Pop}}\right)$ to increase (see Fig. 5B). Thus an increase of inhibition always leads to an increase of spiking variability. An increase of external excitation to the excitatory neurons (via $r_{\varepsilon \varepsilon}^{\mathrm{ext}}$ ), on the other hand, leads to a decrease of $\mathrm{CV}_{s}^{\text {pop }}$.

To demonstrate these effects analytically we derived $\mathrm{CV}^{\mathrm{pop}}$ for a network of PIF model neurons using Eqs. 26-28, where we obtained the steady-state spike rate of the inhibitory neurons, $r^{\mathrm{pop}}$, analogously to $r^{\mathrm{pop}}$ (as described above). Below, we express $\mathrm{CV}_{\varepsilon}^{\mathrm{pop}}$ as a function of either $r_{\tau \varepsilon}^{\mathrm{ext}}, J_{\tau \varepsilon}^{\mathrm{rec}}$, or $J_{\varepsilon \tau}^{\mathrm{rec}}$, and lump together all other fixed parameters in a number of constants,

$$
\mathrm{CV}_{\mathcal{E}}^{\mathrm{pop}}=\left\{\begin{array}{l}
\left(c_{1} r_{\mathcal{I E}}^{\mathrm{ext}}+c_{2}\right) /\left(c_{3}-c_{4} r_{\mathcal{I E}}^{\mathrm{ext}}\right) \\
c_{5} J_{\mathcal{I E}}^{\mathrm{rec}}+c_{6} \\
\left(c_{7}\left(J_{\mathcal{E}}^{\mathrm{rec}}\right)^{2}-c_{8} J_{\mathcal{E} \mathcal{I}}^{\mathrm{rec}}\right) /\left(c_{9}+c_{10} J_{\mathcal{E} \mathcal{I}}^{\mathrm{rec}}\right) .
\end{array}\right.
$$

The constants $c_{1}, \ldots, c_{10}$ in $E q .39$ are nonnegative functions of the fixed parameters. Clearly, an increase of $r_{\tau \varepsilon}^{\mathrm{ext}}$ or the magnitudes of $J_{\mathcal{T S}}^{\mathrm{rec}}$ and $J^{\mathrm{rec}}$ each produce an increase of $\mathrm{CV}_{\mathcal{S}}^{\mathrm{pop}}$ (since $J_{s T}^{\mathrm{rec}}<0$ ). Considering a single PIF neuron receiving (external) excitatory and inhibitory input for comparison, we use Eq. 37 with mean $\mu$ and standard deviation $\sigma$ of the input given by Eqs. 5 and 6, respectively, to express the $\mathrm{CV}$ as

$$
\mathrm{CV}=\sqrt{\frac{J_{\mathcal{E}}^{2} K_{\mathcal{E}} r_{\mathcal{E}}+J_{\mathcal{I}}^{2} K_{\mathcal{I}} r_{\mathcal{I}}}{\Delta V\left(J_{\mathcal{E}} K_{\mathcal{E}} r_{\mathcal{E}}+J_{\mathcal{I}} K_{\mathcal{I}} r_{\mathcal{I}}\right)} .}
$$
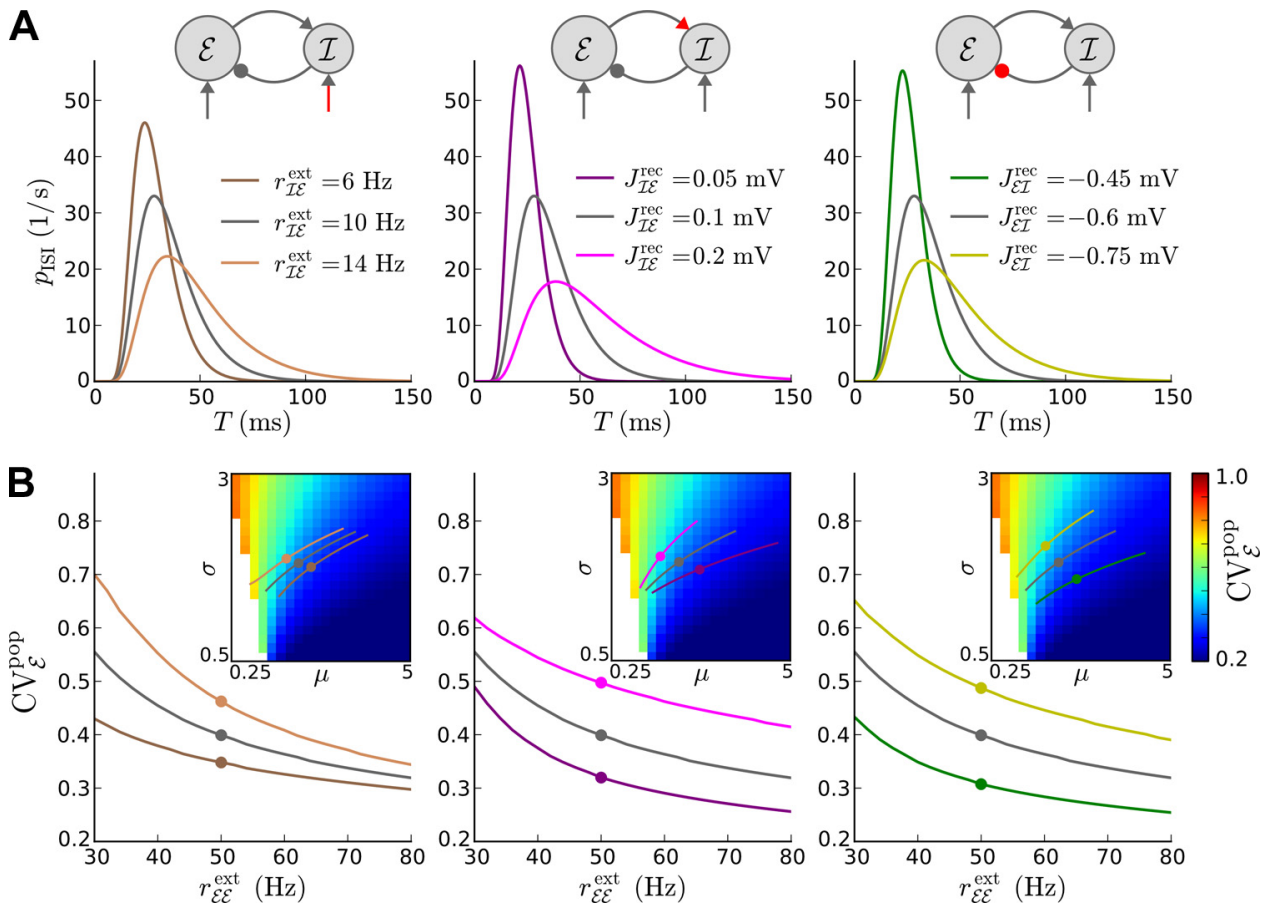

$J$ Neurophysiol $\cdot$ doi:10.1152/jn.00586.2013 • www.jn.org
Fig. 5. Changes of spiking variability caused by network interaction. A: ISI distributions $\left(p_{\text {ISI }}\right)$ of excitatory aEIF neurons for $r_{\mathcal{E}}^{\text {ext }}=$ $50 \mathrm{~Hz} . J_{\mathcal{s T}}^{\mathrm{rec}}=-0.6 \mathrm{mV}, r_{\mathcal{T E}}^{\mathrm{ext}}=10 \mathrm{~Hz}$, and $J_{\mathcal{T E}}^{\mathrm{rec}}=0.1 \mathrm{mV}$ if not indicated otherwise. $B$ : ISI CV for excitatory neurons ( $\left.\mathrm{CV}^{\text {pop }}\right)$ as a function of $r_{\mathcal{E E}}^{\text {ext }}$. Color code as in $A$. Dots indicate the input and ISI CV values for the ISI distributions in A. Insets: ISI CV as a function of the input parameters $\mu$ and $\sigma$ for the excitatory neurons. Lines and dots (insets) correspond to those of equal color in $B$. 
A

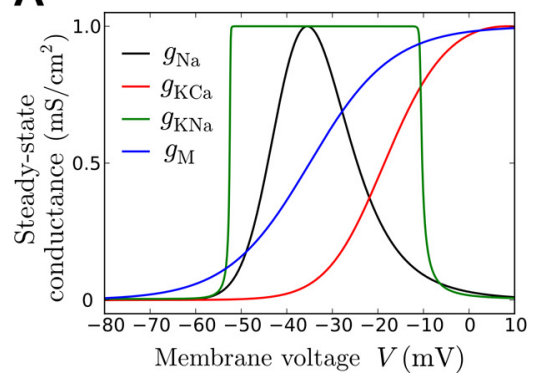

B

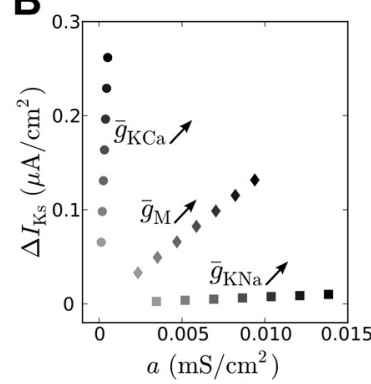

C

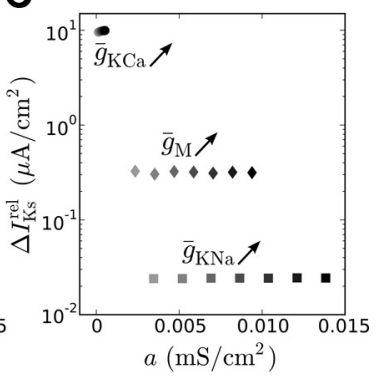

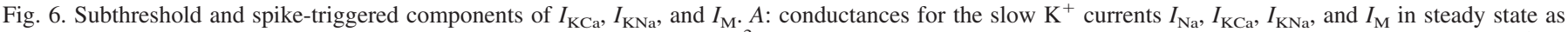

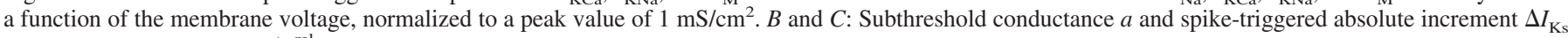

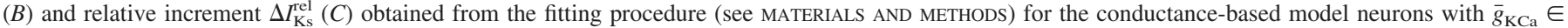

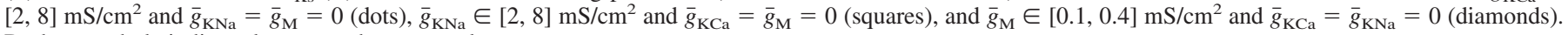
Darker symbols indicate larger conductance values.

Note that Eq. 40 is only valid for positive mean input $\left(J_{\mathcal{E}} K_{\mathcal{E}} r_{\mathcal{E}}+\right.$ $J_{\mathcal{I}} K_{\mathcal{I}} r_{\mathcal{I}}>0$ ). Again, ISI variability increases with inhibition. The effect of inhibition on spiking variability can be understood intuitively as follows. Inhibitory synaptic input reduces the mean total synaptic input $\mu$ and increases its standard deviation $\sigma$ for the target neuron (population), which in turn causes an increase of ISI variability.

Subthreshold and spike-triggered components of slow $\mathrm{K}^{+}$ currents. Here we examine how the two types of an adaptation current in the aEIF model reflect different slow $\mathrm{K}^{+}$currents in a detailed conductance-based neuron model. First, we consider three prominent slow $\mathrm{K}^{+}$currents: a $\mathrm{Ca}^{2+}$-activated afterhyperpolarization current $\left(I_{\mathrm{KCa}}\right)$, a $\mathrm{Na}^{+}$-activated current $\left(I_{\mathrm{KNa}}\right)$ and the voltage-dependent $\mathrm{M}$ current $\left(I_{\mathrm{M}}\right)$. Figure $6 \mathrm{~A}$ shows how the conductances associated with these $\mathrm{K}^{+}$currents depend on the membrane voltage in the steady state, compared with the steady-state spike-generating $\mathrm{Na}^{+}$conductance. The threshold membrane voltage at which a spike is elicited in response to a slowly increasing input current is primarily determined by the conductance-voltage relationship for $\mathrm{Na}^{+}$. The threshold value lies in the interval where this curve has a positive slope (the precise value depends on the peak conductances of all currents and on the input). The curve $g_{\mathrm{Na}, \infty}(V)$ thus indicates the subthreshold and suprathreshold membrane volt-
A components of a range of slow $\mathrm{K}^{+}$currents. $A$ : steady-state $\mathrm{K}^{+}$conductance $g_{\mathrm{Ks}, \infty}(V)=$ $\bar{g}_{\mathrm{Ks}} \omega_{\infty}(V)$ as a function of the membrane voltage, for the generic Hodgkin-Huxleytype description of a slow $\mathrm{K}^{+}$current (see MATERIALS AND METHODS), with half-activation voltage $\alpha=-40 \mathrm{mV}$ (left curves); $\alpha=$ $-10 \mathrm{mV}$ (right curves); inverse steepness $\beta=6,9$, and $12 \mathrm{mV}$; and peak conductance $\bar{g}_{\mathrm{Ks}}=1 \mathrm{mS} / \mathrm{cm}^{2}$. The dashed curve indicates the $\mathrm{Na}^{+}$conductance $g_{\mathrm{Na}, \infty}(V)$ of the conductance-based model, normalized to a maximum value of $1 \mathrm{mS} / \mathrm{cm}^{2} . B$ : subthreshold conductance $a$ obtained from the fitting procedure for different values of the parameters $\alpha$ and $\beta$. C: absolute and relative spiketriggered increments $\Delta I_{\mathrm{Ks}}$ (top) and $\Delta I_{\mathrm{Ks}}^{\mathrm{rel}}$ (bottom), respectively, as a function of $\alpha$, for $\tau_{\omega}=100 \mathrm{~ms}($ left $)$ and $\tau_{\omega}=300 \mathrm{~ms}($ right $)$.
Fig. 7. Subthreshold and spike-triggered age ranges. In the subthreshold voltage range the conductance $g_{\mathrm{KCa}, \infty}$ is almost zero, while the conductances $g_{\mathrm{KNa}, \infty}$ and $g_{\mathrm{M}, \infty}$ reach significant values close to the voltage threshold. Thus the curves in Fig. $6 A$ indicate that $I_{\mathrm{KCa}}$ is activated by spikes, while $I_{\mathrm{M}}$ and particularly $I_{\mathrm{KNa}}$ can be increased in the absence of spiking.

The results of the fitting procedure in Fig. 6, $B$ and $C$, show the absolute and relative amounts of current triggered by a spike vs. its subthreshold level quantified by the voltage independent conductance $a$. $I_{\mathrm{KCa}}$ has a dominant spike-triggered component as expected, while $I_{\mathrm{KNa}}$ shows a very small increment caused by a spike compared with the subthreshold component. $I_{\mathrm{M}}$, on the other hand, shows significant levels of both components. Note, however, that the amount of $I_{\mathrm{M}}$ elicited by a spike is smaller compared with the level of $I_{\mathrm{M}}$ that can be caused by subthreshold membrane depolarization without spiking (since $\Delta I_{\mathrm{Ks}}^{\mathrm{rel}}<1$ for $I_{\mathrm{Ks}} \equiv I_{\mathrm{M}}$, see Fig. $6 C$ ).

We further considered a range of biologically plausible slow $\mathrm{K}^{+}$currents. That is, we varied the steady-state conductancevoltage relationship for $\mathrm{K}^{+}, g_{\mathrm{Ks}, \infty}(V)$, within a realistic range, as shown in Fig. $7 A$, and quantified the subthreshold and spike-triggered components for each of these $\mathrm{K}^{+}$currents (see Fig. 7, $B$ and $C$ ). The value of subthreshold conductance $a$ naturally increases with the fraction of $\mathrm{K}^{+}$conductance present
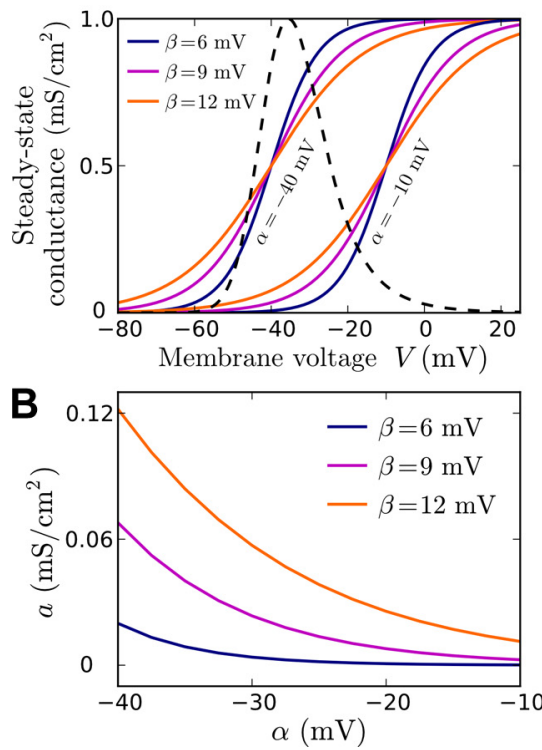

C
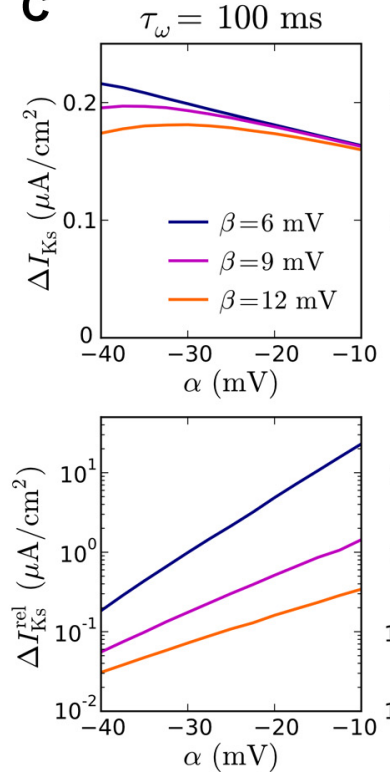
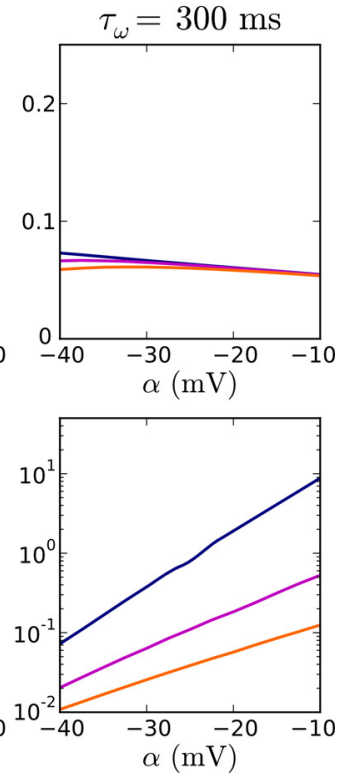
at subthreshold voltage values. For the quantification of spiketriggered current increments we also considered different $\mathrm{K}^{+}$ time constants $\tau_{\omega}$. The absolute value of current increment $\Delta I_{\mathrm{Ks}}$ decreases with increasing $\tau_{\omega}$ and changes only slightly with changes of the shape of the conductance-voltage curve $g_{\mathrm{Ks}, \infty}(V)$ (via the parameters $\alpha, \beta$ ). However, the current increment caused by a spike relative to the amount of current already present in the absence of spiking $\left(\Delta I_{\mathrm{Ks}}^{\mathrm{rel}}\right)$ is strongly determined by $g_{\mathrm{Ks}}(V) . \Delta I_{\mathrm{Ks}}^{\text {rel }}$ increases with an increase of half-activation voltage (parameter $\alpha$ ), steepness (via parameter $\beta)$ and with decreasing time constant $\left(\tau_{\omega}\right)$.

Effects of slow $K^{+}$currents on I-O curve and ISI variability. Here we examine how the different types of slow $\mathrm{K}^{+}$current affect the I-O curve and spiking variability of uncoupled conductance-based model neurons subject to noisy inputs and compare the effects to those caused by subthreshold and spike-triggered adaptation in aEIF neurons. Without a slow $\mathrm{K}^{+}$ current, the spike rate I-O curve does not change over time (see Fig. 8A). An increase of $I_{\mathrm{KCa}}$ has a purely divisive effect on the I-O curve while an increase of $I_{\mathrm{M}}$ changes this curve in a mostly subtractive and slightly divisive way. For both types of slow $\mathrm{K}^{+}$current the adapting spike rates reach their steadystate values in $<500 \mathrm{~ms}$. These effects are consistent with our results based on the aEIF model, given that $I_{\mathrm{KCa}}$ predominantly depends on spikes and $I_{\mathrm{M}}$ includes both, subthreshold as well as spike-triggered, components (Fig. $6 B$ ). In case of increased $I_{\mathrm{KNa}}$, on the other hand, the steady-state I-O curve is significantly altered in both ways (subtractively and divisively), and the spike rate adapts very slowly, that is, steady-state rates are reached after several seconds. At first sight, this seems contradictory to the effect predicted above for subthreshold adaptation, considering that the amount of $I_{\mathrm{KNa}}$ triggered by a spike is small compared with its subthreshold level. Since the timescale of $I_{\mathrm{KNa}}$ is very large (Fig. $8 A$ and Wang et al. 2003) even a small spike-triggered component leads to a significant divisive change of the steady-state I-O curve, cf. Eq. 36. This divisive effect is caused by $\mathrm{K}^{+}$current building up slowly because of small current increments triggered repeatedly by repetitive spiking and very slow decay between spikes due to the large timescale of the current.

Considering ISI variability, an increase of $I_{\mathrm{KCa}}$ reduces the $\mathrm{CV}$ for small values of mean input $\mu$ and increases the $\mathrm{CV}$ for larger values of $\mu$ (see Fig. $8 B$ ). An increase of each of the other slow $\mathrm{K}^{+}$currents, $I_{\mathrm{KNa}}$, and $I_{\mathrm{M}}$, leads to an increase of ISI $\mathrm{CV}$ in general. These effects are consistent with those caused by subthreshold and spike-triggered adaptation currents in the aEIF model, considering the subthreshold and spike-triggered components of $I_{\mathrm{KCa}}, I_{\mathrm{KNa}}$, and $I_{\mathrm{M}}$, respectively (Fig. 6). Thus, the results from the detailed conductance-based neuron model are in agreement with the results based on the adaptive IF models presented above.

\section{DISCUSSION}

In this study, we have systematically examined how adaptation currents and synaptic inhibition modulate the threshold and gain of spiking as well as ISI variability in response to fluctuating inputs resulting from stochastic synaptic events. Based on a simple neuron model with subthreshold and spiketriggered adaptation components, we used analytical and numerical tools to describe spike rates and ISIs for a wide range of input statistics. We then measured subthreshold and spiketriggered components of different types of a slow $\mathrm{K}^{+}$current using detailed conductance-based model neurons, and we val-
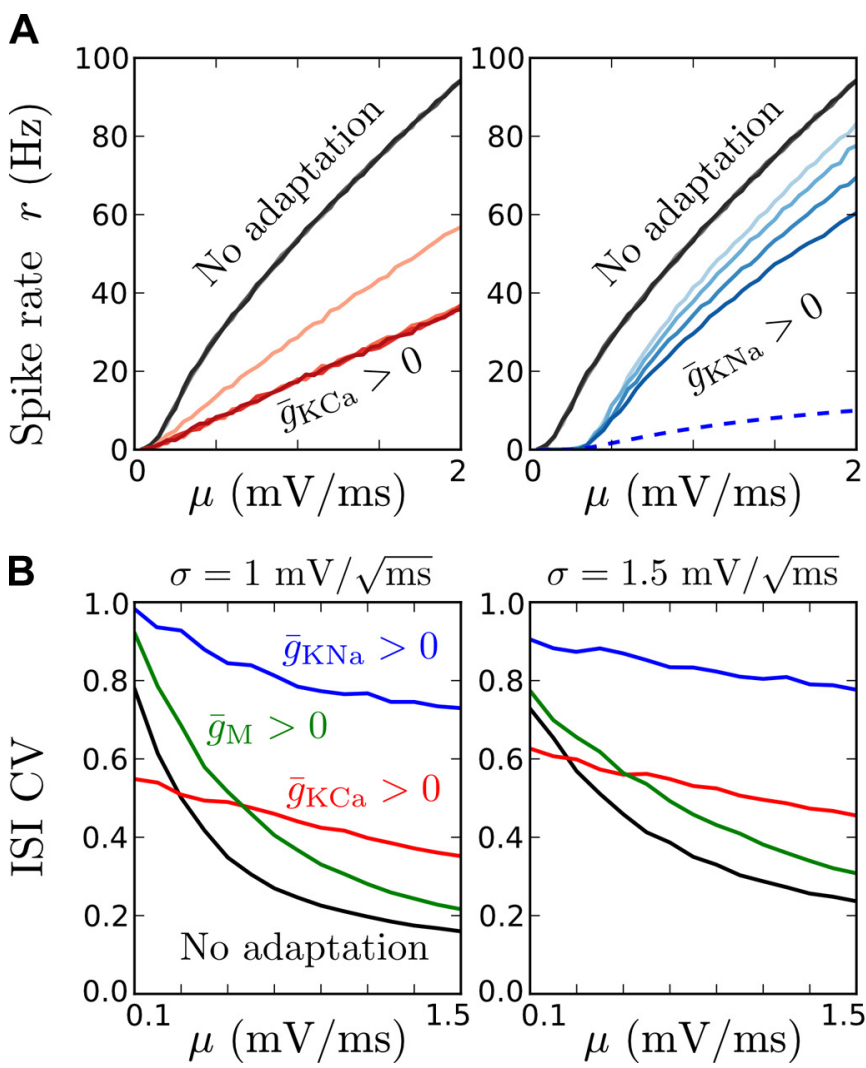
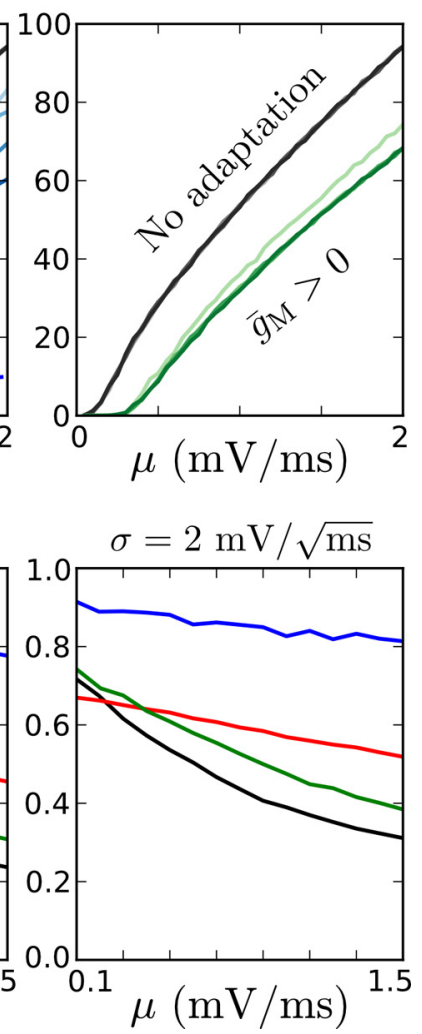

Fig. 8. Effects of $I_{\mathrm{KCa}}, I_{\mathrm{KNa}}$, and $I_{\mathrm{M}}$ on I-O curve and ISI variability. $A$ : spike rate of a conductance-based model neuron without slow $\mathrm{K}^{+}$currents, $\bar{g}_{\mathrm{KCa}}=\bar{g}_{\mathrm{KNa}}=\bar{g}_{\mathrm{M}}=0$ (black), and with either type of slow $\mathrm{K}^{+}$ current included, $\bar{g}_{\mathrm{KCa}}=8 \mathrm{mS} / \mathrm{cm}^{2}$ (red), $\bar{g}_{\mathrm{KNa}}=8 \mathrm{mS} / \mathrm{cm}^{2}$ (blue), $\bar{g}_{\mathrm{M}}=0.4 \mathrm{mS} / \mathrm{cm}^{2}$ (green), in response to a sudden increase of mean input $\mu$, measured in four subsequent time intervals of $250 \mathrm{~ms}$ after that increase (light to dark colors). The baseline mean input was $\mu=0.05 \mathrm{mV} / \mathrm{ms}$ and the input standard deviation was $\sigma=0.5 \mathrm{mV} / \sqrt{\mathrm{ms}}$. Average values over 50 independent trials are shown. The adapting I-O curve of the neuron with increased $I_{\mathrm{KNa}}\left(\bar{g}_{\mathrm{KNa}}=8 \mathrm{mS} /\right.$ $\mathrm{cm}^{2}$ ) converges very slowly to the steadystate curve (dashed blue) measured $20 \mathrm{~s}$ after the increase in $\mu$. B: ISI CV of the neurons in $\mathrm{A}$ as a function of mean input $\mu$ for low (left), medium (middle), and high (right) noise intensity $(\sigma=1,1.5$, and $2 \mathrm{mV} /$ $\sqrt{\mathrm{ms}})$, respectively. The ISIs were collected over an interval of $10 \mathrm{~s}$ after the steady-state spike rates were reached, in 50 independent trials. 
idated our (analytical) results from the simple neuron model by numerical simulations of the detailed model.

We have shown that a purely subthreshold voltage-dependent adaptation current increases the threshold for spiking and reduces the gain at low spike rates in the presence of input fluctuations. This type of current produces a long-tailed ISI distribution and thus leads to an increase of variability for a broad range of input statistics. A spike-triggered adaptation current, on the other hand, causes a divisive change of the I-O curve, thereby reducing the response gain but leaving the response threshold unaffected, irrespective of the input noise intensity. This type of current decreases the ISI CV for fluctuation-dominated inputs but increases the $\mathrm{CV}$ when the mean input is strong, i.e., it reduces the sensitivity of spiking variability to the mean input. For comparison, an increase of external inhibition leads to a subtractive shift of the I-O curve while an increase of recurrent inhibition changes it divisively. The ISI variability, however, is increased by both types of synaptic inhibition.

We have further demonstrated that the $\mathrm{Ca}^{2+}$-activated afterhyperpolarization $\mathrm{K}^{+}$current is effectively captured by a simple description based on spike-triggered increments, while the muscarine-sensitive and $\mathrm{Na}^{+}$-activated $\mathrm{K}^{+}$currents, respectively, have dominant subthreshold components. Despite its small spike-triggered component, the $\mathrm{Na}^{+}$-dependent $\mathrm{K}^{+}$ current also substantially affects the neuronal gain, due to its large timescale.

Methodological aspects. Our approach involves the diffusion approximation and Fokker-Planck equation, both of which have been widely applied to analyze the spike rates of scalar IF type neurons in a noisy setting (see, e.g., Amit and Brunel 1997; Brunel 2000; Fourcaud-Trocmé et al. 2003; Burkitt 2006; Roxin et al. 2011). Our assumption of separated timescales between slow adaptation and fast membrane voltage dynamics has also been frequently used in such a setting (Brunel et al. 2003; La Camera et al. 2004; Gigante et al. 2007b; Richardson 2009; Augustin et al. 2013). While most of these previous studies concentrated on spike rate dynamics, here we focused on asynchronous (nonoscillatory) activity. To examine ISI distributions we extended the method described previously for scalar IF models, which is based on the first passage time problem (Tuckwell 1988; Ostojic 2011), to the aEIF model, accounting for the dynamics of the adaptation current between spikes. Furthermore, we analytically derived an expression for the steady-state spike rate (i.e., steady-state I-O relationship) based on Brunel et al. (2003) and an approximation of the ISI CV using recent results from Urdapilleta (2011) for the perfect IF model with two types of an adaptation current (aPIF model). The I-O functions we calculated can be used to relate (adaptive) spiking neuron models to linearnonlinear cascade models, which describe the instantaneous spike rate of a neuron by applying to the stimulus signal successively a linear temporal filter and a static nonlinear function (Ostojic and Brunel 2011). Such cascade models have proven valuable for studying how sensory inputs are mapped to neuronal activity (see, e.g., Schwartz et al. 2006; Pillow et al. 2008).

It is worth noting that our approach further allows to easily calculate the power spectrum $\mathcal{P}$ and (normalized) autocorrelation function $\mathcal{A}$ of the neuronal spike train once the ISI distribution has been obtained, via the relation

$$
\mathcal{P}(\omega)=\hat{\mathcal{A}}(\omega)=r_{\infty} \operatorname{Re}\left(\frac{1+\hat{p}_{\text {ISI }}(\omega)}{1-\hat{p}_{\text {ISI }}(\omega)}\right),
$$

where $\hat{\mathcal{A}}$ and $\hat{p}_{\text {ISI }}$ ISI denote the Fourier transforms of the autocorrelation function and ISI distribution, respectively (see Gerstner and Kistler 2002). Equation 41 strictly applies to memoryless (so-called renewal) stochastic processes and an adaptation mechanism usually leads to a violation of this requirement for a model neuron subject to fluctuating input. Here we have derived a renewal process $\left(V_{i}(t), \bar{w}(t)\right)$ from the original nonrenewal process $\left(V_{i}(t), w(t)\right)$ by averaging the adaptation current and self-consistently determining its reset value (see ISI distribution). An alternative approach that allows for the application of the above relationship Eq. 41 to adapting model neurons has recently been described in Naud and Gerstner (2012).

Modulation of spike rate threshold and gain. Purely subtractive and divisive changes of the I-O curve by subthreshold and spike-triggered adaptation, respectively, have previously been shown for model neurons considering constant current inputs but neglecting input fluctuations (Prescott and Sejnowski 2008; Ladenbauer et al. 2012). These theoretical results describe the effects shown in recent in vitro experiments that involved blocking the low-threshold $\mathrm{M}$ current and a $\mathrm{Ca}^{2+}$-activated $\mathrm{K}^{+}$ current separately [Deemyad et al. 2012 (Fig. 3); see also Alaburda et al. 2002 (Fig. 3), Smith et al. 2002, and Miles et al. 2005 (Fig. 1) for experimental evidence of either effect]. Here we have shown that a subthreshold adaptation current also causes a reduction of response gain (in addition to an increase of response threshold) when the fluctuations of the input are strong compared with its mean. On the other hand, a spiketriggered adaptation current decreases the response gain over the whole input range, irrespective of the level of input fluctuations. These results apply for adapting as well as the adapted (steady) states. ${ }^{2}$ When considering the onset I-O curve, i.e., the immediate response to a sudden increase of input, an increased level of spike-triggered adaptation current due to preadaptation has been shown to produce a rather subtractive change (Benda et al. 2010). This, however, does not contradict our results. On the contrary, either type of adaptation current (subthreshold or spike-triggered) naturally leads to a subtractive change of the onset I-O curve for neurons which are preadapted to an increased input (not shown).

Notably, when considering conductance-based noisy synaptic inputs, an increase in balanced synaptic background activity can also reduce the spike rate gain (Chance et al. 2002; Burkitt et al. 2003) and external inhibition can reduce the gain and increase the response threshold at the same time (Mitchell and Silver 2003). This means that the response gain can change due to external inputs that are independent of the activity of the target neuron, which can be understood as follows. An increase of noisy (excitatory or inhibitory) synaptic conductance leads to an increase of total membrane conductance, which causes a purely subtractive change of the I-O curve, and an increase in synaptic current noise, which causes an additive change of the I-O curve and decreases its slope (particularly for small input

\footnotetext{
${ }^{2}$ Note that in case of a very large adaptation timescale a (small) spiketriggered adaptation current has a negligible effect on the adapting I-O curve, evaluated shortly after the input steps, but a significant effect on the steadystate I-O curve (see Fig. $8 A$ ).
} 
strengths) (Chance et al. 2002) (Fig. 3). Both effects combined lead to the observed change of response gain. The two separate components are included in our results. An increase of membrane conductance (represented by $g_{\mathrm{L}}$ in the aEIF model) subtracts from the spike rate response, see $E q .18$, and the abovementioned effects of an increase of noise intensity $\sigma$ have been described in RESUlts (see Fig. 2D).

Modulation of response gain is an important phenomenon, particularly in sensory neurons, because neuronal sensitivity to changes in the input is amplified or downscaled without changing input selectivity. A spike-dependent adaptation current thus represents a cellular mechanism by which this is achieved. For example, neuronal response gain increases during selective attention (McAdams and Maunsell 1999). It has been shown in vivo that the neuromodulator acetylcholine $(\mathrm{ACh})$ contributes substantially to attentional upregulation of spike rates (Herrero et al. 2008). Cholinergic changes of neuronal excitability and response gain (Soma et al. 2012) in turn are likely produced via downregulation of slow $\mathrm{K}^{+}$currents (Madison et al. 1987; McCormick 1992; Sripati and Johnson 2006). Together with our results, these observations suggest that excitability and response gain of cortical neurons are controlled by neuromodulatory substances through (de)activation of subthreshold and spike-triggered $\mathrm{K}^{+}$currents, respectively.

We have further shown that external inhibitory synaptic inputs change the I-O curve subtractively, which is consistent with the results of a previous numerical study using a conductance based neuron model without consideration of noise (Capaday 2002). Recurrent synaptic (feedback) inhibition, which is a function of the neuronal spike rate, on the other hand, reduces the response gain. This is in agreement with the results obtained by (Sutherland et al. 2009) based on IF type neurons subject to noisy inputs. Recent in vivo recordings from mouse visual cortex have shown that distinct types of inhibitory neurons produce these differential effects (i.e., subtractive and divisive changes of I-O curves) at their target neurons (Wilson et al. 2012). Functional connectivity analysis suggests that the inhibitory neurons that changed the I-O curve of their target neurons subtractively were less likely connected recurrently to the recorded targets than the inhibitory neurons that changed the responses of the targets divisively (Wilson et al. 2012). By application of our results based on the simple network model, the observed differential effects caused by the two types of inhibitory cells can thus be explained by their patterns of connectivity with the target cells.

Effects on ISI variability. We have shown that a spiketriggered adaptation current reduces high ISI variability at low spike rates (when input fluctuations are strong compared with the mean) and increases low ISI variability at high spike rates (caused by a large mean input). This result is in agreement with a previous numerical simulation study (Liu and Wang 2001) but seems to disagree with other theoretical work (Wang 1998; Prescott and Sejnowski 2008; Schwalger et al. 2010) at first sight. Wang (1998) and later Prescott and Sejnowski (2008) showed that spike-driven adaptation reduces the ISI CV at low spike rates but they did not find an increase of ISI CV at higher spike rates in their simulation studies. The reason for this is that the ISI CVs of adapting and nonadapting neurons were compared at equal spike rates (i.e., at equal mean ISIs) but different input statistics. That is, the input to the adapting neurons was adjusted to compensate for the change of spike rate (or mean ISI) caused by the adaptation currents. Increasing the mean input to the adapting neurons to achieve equal mean ISIs, however, decreases its ISI CV (cf. Eq. 37). Here we compare the ISI statistics across different neurons for equal inputs. On the other hand, Schwalger et al. (2010) analyzed the ISI statistics of perfect IF model neurons with spike-triggered adaptation and found that this type of adaptation always leads to an increase of ISI CV in response to a noisy input current. Their approach is similar to the one presented here but differs in that the dynamics of the adaptation current was neglected in Schwalger et al. (2010); see Fig. 1B, bottom, for a visualization of that difference. Assuming a stationary adaptation current leads to a reduced effective mean input to the neuron, leaving the input variance unchanged, which always causes increased ISI variability (cf. Eq. 37). Together with theoretical work showing that a spike-dependent adaptation current causes negative serial ISI correlations (Prescott and Sejnowski 2008; Farkhooi et al. 2011), our results suggest that spike rate coding is improved by such a current for low-frequency inputs (Prescott and Sejnowski 2008; Farkhooi et al. 2011).

In contrast, an adaptation current that is predominantly driven by the subthreshold membrane voltage usually leads to an increase of ISI CV, as we have demonstrated. This seems to be not consistent with a previous study (Prescott and Sejnowski 2008) where subthreshold adaptation was found to produce a small decrease of ISI variability. The apparent discrepancy is caused by differences in the presentation of the data: Prescott and Sejnowski (2008) compared the ISI CVs for equal spike rates as explained above. That is, the mean input was adjusted to obtain equal mean ISIs for adapting and nonadapting neurons but the input variance remained unchanged. However, increasing the mean input ( $\mu$ in $E q$. 37) to the adapting neuron counteracts the effect of subthreshold adaptation on the effective mean input ( $\mu_{a}$ in Eq. 37). Consequently, one cannot observe an increased ISI CV in neurons with subthreshold adaptation currents when the mean input to these neurons is increased. Note that our results do not contradict those in (Prescott and Sejnowski 2008) but instead reveal that an increase of a subthreshold adaptation current always causes an increase of ISI CV for given input statistics and an increase of a spike-dependent adaptation current leads to an increase of ISI $\mathrm{CV}$ if the mean input is large.

Finally, we have shown that an increase in synaptic inhibition increases the ISI variability, regardless of whether this inhibition originates from an external population of neurons or from recurrently coupled ones. An intuitive explanation for this effect is that increased inhibitory input reduces the mean input but increases the input variance (see Eqs. 5 and 6 ). The reason why recurrent synaptic inhibition and spike-triggered adaptation change the ISI variability in opposite ways in a fluctuation-dominated input regime could be the different timescales. Synaptic inhibition usually acts on a much faster timescale than adaptation currents whose time constants range from about 100 $\mathrm{ms}$ to seconds. Thus recurrent synaptic inhibition in contrast to spike-triggered adaptation cannot provide a memory trace of past spiking activity (over a duration of several ISIs) that could shape the ISI distribution. Notably, our results on ISIs in a network setting strictly apply to networks in asynchronous states. Recurrent synaptic inhibition, however, can also mediate oscillatory activity (Brunel 2000; Brunel et al. 2003; 
Isaacson and Scanziani 2011; Augustin et al. 2013) where the variability of ISIs might be affected differently.

\section{ACKNOWLEDGMENTS}

We thank Maziar Hashemi-Nezhad for helpful comments on the manuscript.

\section{GRANTS}

This work was supported by Deutsche Forschungsgemeinschaft in the framework of Collaborative Research Center SFB910.

\section{DISCLOSURES}

No conflicts of interest, financial or otherwise, are declared by the author(s).

\section{AUTHOR CONTRIBUTIONS}

Author contributions: J.L. and K.O. conception and design of research; J.L. and M.A. performed experiments; J.L. and M.A. analyzed data; J.L., M.A., and K.O. interpreted results of experiments; J.L. and M.A. prepared figures; J.L. drafted manuscript; J.L., M.A., and K.O. edited and revised manuscript; J.L., M.A., and K.O. approved final version of manuscript.

\section{REFERENCES}

Adams PR, Brown DA, Constanti A. Pharmacological inhibition of the M-current. J Physiol 332: 223-262, 1982.

Alaburda A, Perrier JF, Hounsgaard J. An M-like outward current regulates the excitability of spinal motoneurones in the adult turtle. $J$ Physiol 540: 875-881, 2002.

Amit DJ, Brunel N. Model of global spontaneous activity and local structured activity during delay periods in the cerebral cortex. Cereb Cortex 7: 237-252, 1997.

Augustin M, Ladenbauer J, Obermayer K. How adaptation shapes spike rate oscillations in recurrent neuronal networks. Front Comput Neurosci 7 : 9, 2013.

Badel L, Lefort S, Brette R, Petersen CCH, Gerstner W, Richardson MJ. Dynamic I-V curves are reliable predictors of naturalistic pyramidal-neuron voltage traces. J Neurophysiol 99: 656-666, 2008.

Benda J, Herz AV. A universal model for spike-frequency adaptation. Neural Comput 15: 2523-2564, 2003.

Benda J, Longtin A, Maler L. Spike-frequency adaptation separates transient communication signals from background oscillations. J Neurosci 25: 23122321, 2005.

Benda J, Maler L, Longtin A. Linear versus nonlinear signal transmission in neuron models with adaptation currents or dynamic thresholds. J Neurophysiol 104: 2806-2820, 2010.

Brette R, Gerstner W. Adaptive exponential integrate-and-fire model as an effective description of neuronal activity. J Neurophysiol 94: 3637-3642, 2005.

Brown DA, Adams PR. Muscarinic suppression of a novel voltage-sensitive $\mathrm{K}^{+}$current in a vertebrate neurone. Nature 283: 673-676, 1980.

Brown DA, Griffith WH. Calcium-activated outward current in voltageclamped hippocampal neurones of the guinea-pig. J Physiol 337: 287-301, 1983

Brunel N. Dynamics of sparsely connected networks of excitatory and inhibitory spiking neurons. J Comput Neurosci 8: 183-208, 2000.

Brunel N, Hakim V, Richardson M. Firing-rate resonance in a generalized integrate-and-fire neuron with subthreshold resonance. Phys Rev E Stat Nonlin Soft Matter Phys 67: 051916, 2003.

Burkitt AN. A review of the integrate-and-fire neuron model: I. Homogeneous synaptic input. Biol Cybern 95: 1-19, 2006.

Burkitt AN, Meffin H, Grayden DB. Study of neuronal gain in a conductance-based leaky integrate-and-fire neuron model with balanced excitatory and inhibitory synaptic input. Biol Cybern 89: 119-125, 2003.

Capaday C. A re-examination of the possibility of controlling the firing rate gain of neurons by balancing excitatory and inhibitory conductances. Exp Brain Res 143: 67-77, 2002.

Chance FS, Abbott LF, Reyes AD. Gain modulation from background synaptic input. Neuron 35: 773-782, 2002.
Compte A, Constantinidis C, Tegner J, Raghavachari S, Chafee MV, Goldman-Rakic PS, Wang XJ. Temporally irregular mnemonic persistent activity in prefrontal neurons of monkeys during a delayed response task. $J$ Neurophysiol 90: 3441-3454, 2003.

Deemyad T, Kroeger J, Chacron MJ. Sub- and suprathreshold adaptation currents have opposite effects on frequency tuning. J Physiol 590: 48394858, 2012.

Destexhe A. Self-sustained asynchronous irregular states and up-down states in thalamic, cortical and thalamocortical networks of nonlinear integrateand-fire neurons. J Comput Neurosci 27: 493-506, 2009.

Destexhe A, Rudolph M, Paré D. The high-conductance state of neocortical neurons in vivo. Nat Rev Neurosci 4: 739-751, 2003.

Destexhe A, Rudolph-Lilith M. Neuronal Noise. New York: Springer, 2012.

Ellis LD, Mehaffey WH, Harvey-Girard E, Turner RW, Maler L, Dunn RJ. SK channels provide a novel mechanism for the control of frequency tuning in electrosensory neurons. J Neurosci 27: 9491-9502, 2007.

Farkhooi F, Muller E, Nawrot M. Adaptation reduces variability of the neuronal population code. Phys Rev E Stat Nonlin Soft Matter Phys 83: 050905, 2011.

Fourcaud-Trocmé N, Hansel D, van Vreeswijk C, Brunel N. How spike generation mechanisms determine the neuronal response to fluctuating inputs. J Neurosci 23: 11628-11640, 2003.

Fuhrmann G, Markram H, Tsodyks M. Spike frequency adaptation and neocortical rhythms. J Neurophysiol 88: 761-770, 2002.

Gerstein GL, Mandelbrot B. Random walk models for the spike activity of a single neuron. Biophys $J$ 4: 41-68, 1964.

Gerstner W, Kistler WM. Spiking Neuron Models. Cambridge, UK: Cambridge Univ. Press, 2002.

Gigante G, Del Giudice P, Mattia M. Frequency-dependent response properties of adapting spiking neurons. Math Biosci 207: 336-351, 2007a.

Gigante G, Mattia M, Del Giudice P. Diverse population-bursting modes of adapting spiking neurons. Phys Rev Lett 98: 148101, 2007b.

Herrero JL, Roberts MJ, Delicato LS, Gieselmann MA, Dayan P, Thiele A. Acetylcholine contributes through muscarinic receptors to attentional modulation in V1. Nature 454: 1110-1114, 2008.

Isaacson JS, Scanziani M. How inhibition shapes cortical activity. Neuron 72: 231-243, 2011.

Jolivet R, Schürmann F, Berger TK, Naud R, Gerstner W, Roth A. The quantitative single-neuron modeling competition. Biol Cybern 99: 417-426, 2008

Kim U, McCormick DA. Functional and ionic properties of a slow afterhyperpolarization in ferret perigeniculate neurons in vitro. J Neurophysiol 80: 1222-1235, 1998.

La Camera G, Rauch A, Lüscher HR, Senn W, Fusi S. Minimal models of adapted neuronal response to in vivo-like input currents. Neural Comput 16: 2101-2124, 2004.

La Camera G, Rauch A, Thurbon D, Lüscher HR, Senn W, Fusi S. Multiple time scales of temporal response in pyramidal and fast spiking cortical neurons. J Neurophysiol 96: 3448-3464, 2006.

Ladenbauer J, Augustin M, Shiau L, Obermayer K. Impact of adaptation currents on synchronization of coupled exponential integrate-and-fire neurons. PLoS Comput Biol 8: e1002478, 2012.

Ladenbauer J, Lehnert J, Rankoohi H, Dahms T, Schöll E, Obermayer K. Adaptation controls synchrony and cluster states of coupled threshold-model neurons. Phys Rev E Stat Nonlin Soft Matter Phys 88: 042713, 2013.

Liu YH, Wang XJ. Spike-frequency adaptation of a generalized leaky integrate-and-fire model neuron. J Comput Neurosci 10: 25-45, 2001.

Madison DV, Lancaster B, Nicoll RA. Voltage clamp analysis of cholinergic action in the hippocampus. J Neurosci 7: 733-741, 1987.

Madison DV, Nicoll RA. Control of the repetitive discharge of rat CA1 pyramidal neurones in vitro. J Physiol 354: 319-331, 1984.

Maimon G, Assad JA. Beyond poisson: increased spike-time regularity across primate parietal cortex. Neuron 62: 426-440, 2009.

Mainen ZF, Sejnowski TJ. Influence of dendritic structure on firing pattern in model neocortical neurons. Nature 382: 363-366, 1996.

McAdams CJ, Maunsell JH. Effects of attention on orientation-tuning functions of single neurons in macaque cortical area V4. J Neurosci 19: 431-441, 1999.

McCormick DA. Neurotransmitter actions in the thalamus and cerebral cortex and their role in neuromodulation of thalamocortical activity. Progr Neurobiol 39: 337-388, 1992.

Miles GB, Dai Y, Brownstone RM. Mechanisms underlying the early phase of spike frequency adaptation in mouse spinal motoneurones. J Physiol 566: 519-532, 2005. 
Mitchell SJ, Silver RA. Shunting inhibition modulates neuronal gain during synaptic excitation. Neuron 38: 433-445, 2003.

Naud R, Gerstner W. Coding and decoding with adapting neurons: a population approach to the peri-stimulus time histogram. PLoS Comput Biol 8: e1002711, 2012.

Naud R, Marcille N, Clopath C, Gerstner W. Firing patterns in the adaptive exponential integrate-and-fire model. Biol Cybern 99: 335-347, 2008.

Ostojic S. Inter-spike interval distributions of spiking neurons driven by fluctuating inputs. J Neurophysiol 106: 361-373, 2011.

Ostojic S, Brunel N. From spiking neuron models to linear-nonlinear models. PLoS Comput Biol 7: e1001056, 2011.

Pillow JW, Shlens J, Paninski L, Sher A, Litke AM, Chichilnisky EJ, Simoncelli EP. Spatio-temporal correlations and visual signalling in a complete neuronal population. Nature 454: 995-999, 2008.

Pospischil M, Piwkowska Z, Bal T, Destexhe A. Comparison of different neuron models to conductance-based post-stimulus time histograms obtained in cortical pyramidal cells using dynamic-clamp in vitro. Biol Cybern 105: 167-180, 2011

Prescott SA, Sejnowski TJ. Spike-rate coding and spike-time coding are affected oppositely by different adaptation mechanisms. J Neurosci 28: 13649-13661, 2008.

Renart A, Brunel N, Wang XJ. Mean-field theory of irregularly spiking neuronal populations and working memory in recurrent cortical networks. In: Computational Neuroscience-A Comprehensive Approach, edited by Feng J. New York: CRC, 425-484, 2004.

Reynolds JH, Heeger DJ. The normalization model of attention. Neuron 61: $168-185,2009$.

Richardson M. Dynamics of populations and networks of neurons with voltage-activated and calcium-activated currents. Phys Rev E Stat Nonlin Soft Matter Phys 80: 1-16, 2009.

Risken H. The Fokker-Planck Equation. New York: Springer, 1996.

Roxin A, Brunel N, Hansel D, Mongillo G, van Vreeswijk C. On the distribution of firing rates in networks of cortical neurons. J Neurosci 31: 16217-16226, 2011.

Sanchez-Vives MV, McCormick DA. Cellular and network mechanisms of rhythmic recurrent activity in neocortex. Nat Neurosci 3: 1027-1034, 2000.

Sanchez-Vives MV, Nowak LG, McCormick DA. Membrane mechanisms underlying contrast adaptation in cat area 17 in vivo. J Neurosci 20: 4267-4285, 2000.
Schwalger T, Fisch K, Benda J, Lindner B. How noisy adaptation of neurons shapes interspike interval histograms and correlations. PLoS Comput Biol 6: e1001026, 2010.

Schwartz O, Pillow JW, Rust NC, Simoncelli EP. Spike-triggered neural characterization. J Vis 6: 484-507, 2006.

Schwindt PC, Spain WJ, Crill WE. Long-lasting reduction of excitability by a sodium-dependent potassium current in cat neocortical neurons. J Neurophysiol 61: 233-244, 1989.

Schwindt PC, Spain WJ, Crill WE. Calcium-dependent potassium currents in neurons from cat sensorimotor cortex. J Neurophysiol 67: 216-226, 1992.

Shadlen MN, Newsome WT. The variable discharge of cortical neurons: implications for connectivity, computation, and information coding. $\mathrm{J} \mathrm{Neu}$ rosci 18: 3870-3896, 1998.

Smith MR, Nelson AB, Du Lac S. Regulation of firing response gain by calcium-dependent mechanisms in vestibular nucleus neurons. J Neurophysiol 87: 2031-2042, 2002.

Soma S, Shimegi S, Osaki H, Sato H. Cholinergic modulation of response gain in the primary visual cortex of the macaque. J Neurophysiol 107: 283-291, 2012.

Sripati AP, Johnson KO. Dynamic gain changes during attentional modulation. Neural Comput 18: 1847-1867, 2006.

Stocker M. $\mathrm{Ca}^{2+}$-activated $\mathrm{K}^{+}$channels: molecular determinants and function of the SK family. Nat Rev Neurosci 5: 758-770, 2004.

Sutherland C, Doiron B, Longtin A. Feedback-induced gain control in stochastic spiking networks. Biol Cybern 100: 475-489, 2009.

Touboul J, Brette R. Dynamics and bifurcations of the adaptive exponential integrate-and-fire model. Biol Cybern 99: 319-334, 2008.

Tuckwell HC. Introduction to Theoretical Neurobiology. Cambridge, UK: Cambridge Univ Press, vol 2, 1988.

Urdapilleta E. Survival probability and first-passage-time statistics of a Wiener process driven by an exponential time-dependent drift. Phys Rev E Stat Nonlin Soft Matter Phys 83: 021102, 2011.

Wang XJ. Calcium coding and adaptive temporal computation in cortical pyramidal neurons. J Neurophysiol 79: 1549-1566, 1998.

Wang XJ, Liu Y, Sanchez-Vives MV, McCormick DA. Adaptation and temporal decorrelation by single neurons in the primary visual cortex. $J$ Neurophysiol 89: 3279-3293, 2003.

Wilson NR, Runyan CA, Wang FL, Sur M. Division and subtraction by distinct cortical inhibitory networks in vivo. Nature 488: 343-348, 2012. 